\title{
Ubiquitylated H2A.Z nucleosomes are associated with nuclear architectural proteins and global transcriptional silencing
}

Marlee K. $\mathrm{Ng}^{1}$, Ulrich Braunschweig ${ }^{2}$, Benjamin J. Blencowe ${ }^{2,3}$ and Peter Cheung $^{1 *}$

${ }^{1}$ Department of Biology, York University, Toronto, ON M3J 1P3, Canada

${ }^{2}$ Donnelly Centre, University of Toronto, Toronto, ON M5S 3E1, Canada

${ }^{3}$ Department of Molecular Genetics, University of Toronto, Toronto, ON M5S 1A8, Canada

*Correspondence: pmcheung@yorku.ca

Keywords

Histone variants, H2A.Z, ubiquitylation, histone modifications, nucleosome, chromatin, epigenetics, transcriptional regulation, ChIP-seq, genome organization 


\section{Summary}

H2A.Z mono-ubiquitylation has been linked to transcriptional repression, but the mechanisms involved are not well understood. To address this, we developed a biotinylation-based approach to purify ubiquitylated H2A.Z (H2A.Zub) mononucleosomes for biochemical and genome-wide analyses. We observe that H2A.Zub nucleosomes are enriched for the repressive histone post-translational modification H3K27me3, but depleted of H3K4 methylation and other modifications associated with active transcription. ChIP-Seq analyses reveal that H2A.Zub-nucleosomes are enriched over non-expressed genes, and suggest that it is the relative ratio of ubiquitylated to non-ubiquitylated H2A.Z, rather than absolute presence or absence of H2A.Z ubiquitylation, that correlates with gene silencing. Finally, we observe that H2A.Zub-eniched mononucleosomes preferentially co-purify with transcriptional silencing factors as well as proteins involved in higher order chromatin organization such as CTCF and cohesin. Collectively, these results suggest an important role for H2A.Z ubiquitylation in mediating global transcriptional repression through its recruitment of silencing factors and nuclear architectural proteins. 


\section{Introduction}

Accessibility of the eukaryotic genome is impacted by how it is packaged into chromatin and how chromatin/chromosomes are looped and folded in 3dimensional space within the nucleus (Bonev and Cavalli, 2016, Van Bortle and Corces, 2012). This organization of the genome regulates many DNA-templated processes including transcription and gene expression. At the simplest level, DNA is wrapped around histone proteins to form nucleosomes, and the 'nucleosome-on-a-string' $10 \mathrm{~nm}$ fiber can further fold into higher-order structures that not only serve to compact the genome, but also form long-range interactions within the genome to assemble functional domains. As the repeating unit of chromatin, each nucleosome is made up of $\sim 147 \mathrm{bp}$ of DNA wrapped around a histone octamer containing 2 copies each of the core histones H2A, H2B, H3 and H4 (Kornberg, 1974). Although most nucleosomes share this same structural architecture, the addition of different histone post-translational modifications (PTMs) on specific sites and histones confer distinct properties and functions to the nucleosomes (Kouzarides, 2007). Common examples of histone PTMs include acetylation, phosphorylation, methylation and monoubiquitylation, and they are deposited or removed by specialized enzymes that either respond to upstream signaling pathways or are constitutively active to maintain the steady-state levels of histone PTMs. The physical and functional effects of histone PTMs vary depending on the type of modification and context, but it is well established that a number of histone PTMs serve to recruit chromatin binding proteins through PTM-dependent interactions (Strahl and Allis, 2000, Jenuwein and Allis, 2001). In such contexts, histone PTMs can serve as signals to target effector proteins to specific regions of the genome, and relay information from upstream signaling pathways to downstream actions (Cheung et al., 2000, Schreiber and Bernstein, 2002). In recent years, it has also been recognized that since nucleosomes contain many different histone PTMs and binding surfaces, the sum of all these binding ligands can together stabilize and regulate interactions between nucleosomes and chromatin binding factors (Ruthenburg et al., 2007, Suganuma and Workman, 2011, Ng and Cheung, 2016). Therefore, determining the combinatorial effects of histone PTMs in the context 
of the nucleosome is critical for our understanding of the mechanisms underlying chromatin-regulated pathways.

In addition to histone PTMs, replacement of core histones with specific histone variants is another important mechanism that adds functional diversity to the nucleosome framework. Histone variants are non-allelic isoforms of core histones and are deposited at strategic positions within the genome to perform specialized functions (Buschbeck and Hake, 2017). For example, H2A.Z is an $\mathrm{H} 2 \mathrm{~A}$-family variant that is often enriched at the nucleosomes flanking transcription start sites (TSSs) of many genes in eukaryotic genomes (Giaimo et al., 2019, Subramanian et al., 2015). With the exception of S. cerevisiae, knockout of the H2A.Z gene in other organisms invariably leads to developmental defects (e.g. in mice) and death (Elgin 1992, Gorovsky 1996, Faast 2001). The specific functions of H2A.Z that are essential for viability are not known, although increasing evidence has demonstrated that H2A.Z is important for transcriptional regulation since its knockdown, or knockdown of the remodeling complexes that deposit H2A.Z to the genome, often leads to dysregulated gene expression (Santisteban et al., 2000, Adam et al., 2001, Mizuguchi et al., 2004, Kobor et al., 2004). H2A.Z ChIP-seq analysis in human cells showed that H2A.Z is enriched at active genes (Barski et al., 2007), suggesting a correlation between H2A.Z and gene activity. H2A.Z has been linked to both transcriptional activation and repression, and these distinct activities are likely mediated through its differential PTMs (e.g. acetylation vs. mono-ubiquitylation, Draker and Cheung, 2009). H2A.Z is acetylated at multiple lysines at its N-terminus by the NuA4 complex in S. cerevisiae or Tip60 in Drosophila and mammals (Keogh et al., 2006, Millar et al., 2006, Kusch et al., 2004, Giaimo et al., 2018). H2A.Z acetylation directly correlates with transcriptional activation (Millar et al., 2006) and expression of mutant H2A.Z that cannot be acetylated disrupts expression of the master regulator $\mathrm{MyoD}$ and downstream differentiation in myoblast cells (Law and Cheung, 2015). Expression of non-acetylatable H2A.Z also blocks transcription of eRNAs and enhancer functions in the context of androgenreceptor $(A R)$-regulated gene activation in prostate cancer cells (Valdes-Mora et al., 2017). In contrast to acetylation, mono-ubiquitylation of H2A.Z at its C- 
terminus has been correlated with transcriptional repression. This modification is mediated by the RING1b E3 ligase of Polycomb Repressor Complex 1 (PRC1) and ubiquitylated H2A.Z is enriched on the transcriptionally silent inactive $X$ chromosome in human female cells (Sarcinella et al., 2007). Ubiquitylated H2A.Z is also found at the promoters of AR-regulated genes prior to hormone induction and transcriptional activation of these genes is accompanied by H2A.Z deubiquitylation, suggesting $\mathrm{H} 2 \mathrm{~A}$.Zub may antagonize transcription initiation (Draker et al., 2011). At present, the exact mechanisms of how H2A.Z by itself, or through H2A.Z acetylation and ubiquitylation, participate in transcriptional regulation are not clearly understood. Early studies in $S$. cerevisiae showed that H2A.Z affects nuclease sensitivity within the genome and it also functions at chromatin boundaries to antagonize heterochromatin spreading (Adam et al., 2001, Meneghini et al., 2003). It is also thought that H2A.Z poises inducible promoters for transcription initiation and lowers the stability of the +1 nucleosome at TSSs to facilitate passage of RNA polymerase II during transcriptional activation (Zhang et al., 2005). Affinity purification studies found that H2A.Z nucleosomes preferentially bind the bromodomain protein Brd2, and the H2A.Z-dependent recruitment of Brd2 to AR-regulated genes is part of the hormone-activated transcription process in prostate cells (Draker et al., 2012). The interaction between H2A.Z and Brd2 has also been reported in melanoma and bladder cancer cells (Vardabasso et al., 2015, Kim et al., 2013). In melanoma cells, the H2A.Z.2 isoform recruits both Brd2 and the transcription factor E2F1 to regulate E2F-target genes. Altogether, these studies suggest that H2A.Zdependent recruitment of interacting proteins, such as Brd2, is a critical mechanistic step in H2A.Z-regulated transcription.

A mechanistic understanding of the role of $\mathrm{H} 2 \mathrm{~A} . \mathrm{Z}$ ubiquitylation has been lacking due to the absence of research tools affording the specific detection and isolation of this modified form of H2A.Z. While multiple antibodies specific for mammalian H2A.Z have been raised, they generally cannot distinguish between non-ubiquitylated and ubiquitylated H2A.Z. Moreover, for some antibodies raised against the C-terminus region where H2A.Z ubiquitylation occurs, their recognition of $\mathrm{H} 2 \mathrm{~A}$.Zub may be blocked due to epitope occlusion by the 
ubiquitin moiety (Sarcinella et al., 2007). Raising antibodies specific for ubiquitylated proteins is confronted by the additional challenge that they would have to recognize the isopeptide junction between the ubiquitin C-terminus and the sequence flanking the ubiquitylated lysine on the target protein. Lastly, H2A.Z can be mono-ubiquitylated at three possible lysine residues (K120, K121 or K125) (Sarcinella et al., 2007, Ku et al., 2012), further complicating the raising of ubiquitylation-specific antibodies.

To advance mechanistic studies of ubiquitylated H2A.Z, we have developed a non-antibody-dependent system that affords its specific detection and purification. This system involves expressing a version of mammalian H2A.Z that auto-biotinylates when it is ubiquitylated, which then allows specific purification of ubiquitylated H2A.Z using streptavidin-coupled reagents. In this study, we demonstrate the efficacy of this system and its utility in biochemical and genome-wide analyses. We also identified a distinct combination of transcription repression-associated histone PTMs that co-exist with H2A.Zub incorporated into nucleosomes. ChIP-sequencing analyses showed that ubiquitylated and non-ubiquitylated H2A.Z overlap throughout the genome, but a relative enrichment of $\mathrm{H} 2 \mathrm{~A}$.Zub over $\mathrm{H} 2 \mathrm{~A} . \mathrm{Z}$ at promoters is associated with transcriptional silencing. Finally, we discovered a number of proteins that preferentially co-purify with ubiquitylated H2A.Z-containing nucleosomes, pointing to possible chromatin modifying enzymes that are recruited by these nucleosomes to mediate transcriptional silencing. Importantly, we observe preferential interactions between CTCF and cohesin components with ubiquitylated H2A.Z, raising the possibility that this modified form of H2A.Z may be involved in the 3D organization of the mammalian genome in conjunction with its role in transcriptional regulation.

\section{Results}

\section{Development and characterization of a ubiquitylation-dependent self- biotinylating system for studying mono-ubiquitylated H2A.Z.}


We previously developed a method named BICON to specifically purify phosphorylated H3 from total chromatin (Lau and Cheung, 2013). That method takes advantage of the highly specific biotinylation of a 15 amino acid sequence (named Avi-tag) by the E. coli biotin ligase BirA when these components are coexpressed in mammalian cells. To adapt that method for studying H2A.Z ubiquitylation, we fused BirA to the C-terminus of H2A.Z, and placed the Avitag on the N-terminus of the ubiquitin molecule (Avi-ub; Fig. 1). We also added a Flag-tag peptide sequence between the H2A.Z and BirA domains to facilitate its detection and purification, and the resulting H2A.Z-Flag-BirA fusion is hereafter termed H2A.Z-FB. By co-expressing H2A.Z-FB and Avi-ub in transfected cells, we reasoned that when H2A.Z-FB is mono-ubiquitylated with Avi-ub, the BirA at its C-terminus would preferentially and automatically biotinylate the Avi-tag on the conjugated ubiquitin since the enzyme and substrate are now physically on the same molecule (see Fig 1A). Therefore, with this system, we are generating a version of $\mathrm{H} 2 \mathrm{~A}$. $\mathrm{Z}$ that self-biotinylates when it is ubiquitylated with Avi-ub.

As a proof of concept, we first co-transfected H2A.Z-FB- and Avi-ub-expression constructs into human $293 \mathrm{~T}$ cells and examined their expression and biotinylation by Western blot analyses (Fig 1B). As an additional control, we generated a non-ubiquitylatable mutant form of H2A.Z-FB whereby the known potential sites of H2A.Z mono-ubiquitylation (K120, K121, and K125) are mutated to arginines (referred to as the K3R3 mutant), and co-expressed that with Avi-ub in 293T cells. Western blot analysis of whole cell extracts or nuclear extracts of the respective transfected cells using anti-Flag antibody showed strong expression of both WT- and K3R3-H2A.Z-FB. As expected, the anti-Flag antibody detected two bands (corresponding to the unmodified H2A.Z-FB and the larger mono-ubiquitylated form of H2A.Z-FB) in lysates from cells transfected with WT H2A.Z-FB, but only the lower molecular weight band in cells expressing the K3R3 mutant. We next examined the presence and detection of biotinylated proteins in the same extracts using horse-radish peroxidaseconjugated avidin (Avidin-HRP) and found that the predominant and most abundant biotinylated protein was indeed the Avi-ub-H2A.Z-FB, which co- 
migrated with the shifted higher molecular weight band detected in the Flag Western blots. The Avidin-HRP-detected band in the WT H2A.Z-FB + Avi-ub sample was not present in the H2A.Z-FB K3R3 mutant + Avi-ub sample, further validating that biotinylation specifically occurs on the Avi-ubiquitylated H2A.ZFB. These results indicate that our experimental system works as predicted and that Avi-ub-H2A.Z-FB is the predominant biotinylated protein in the transfected cells.

We also compared expression of the transfected H2A.Z-FB + Avi-ub combination in several different mammalian cell lines and found that this system works best in human $293 \mathrm{~T}$ cells (data not shown). Therefore, all subsequent biochemical and genomic analyses were performed using this cell line. Furthermore, we found that in mono-nucleosome-enriched fractions (harvested from micrococcalnuclease digested nuclei), in addition to Avi-ub-H2A.Z-FB, Avidin-HRP also detected a small amount of a lower molecular weight band that corresponds to the size of endogenous ubiquitylated H2A.Z (Fig 1C). Since H2A.Z-FB can partner with endogenous H2A.Z (or H2A) in the nucleosome context, it is possible that the H2A.Z-FB also biotinylates other endogenous Avi-ub$\mathrm{H} 2 \mathrm{~A} . \mathrm{Z} / \mathrm{H} 2 \mathrm{~A}$ on the same nucleosome or in close proximity. We note that this lower band is also detected by Avidin-HRP in cells transfected with Flag-NLSBirA (BirA fused to Flag and a nuclear localization signal) control, indicating that the presence of BirA and Avi-ub in cells can result in some background amounts of biotinylation of the Avi-ubiquitylated endogenous histones. However, this background amount is minor compared to the biotinylated Avi-ub-H2A.Z-FB in the H2A.Z-FB + Avi-ub transfected cells. Finally, we found that in the K3R3 H2A.Z-FB + Avi-ub transfected cells, only the lower band is detected by AvidinHRP. In this context, there were higher amounts of the biotinylated lower band than found in WT H2A.Z-FB + Avi-ub transfected cells, suggesting that in the absence of a preferred biotinylation site on the Avi-ub-H2A.Z-FB itself, the BirA on this fusion protein can biotinylate less preferred Avi-tags on nearby Aviubiquitylated H2A.Z/H2A. These findings together show that wild-type H2A.ZFB preferentially and predominantly biotinylates the Avi-tagged ubiquitin on 
itself first, and secondarily biotinylates other nearby Avi-ubiquitylated histones if the main ubiquitylation site on H2A.Z-FB is mutated.

\section{Immunoprecipitation of mono-nucleosomes containing Avi-ubiquitylated} H2A.Z-FB or non-ubiquitylated H2.Z-FB.

Our previous work, as well as studies by others, has shown that epitope- or GFPtagging of $\mathrm{H} 2 \mathrm{~A} . \mathrm{Z}$ at the $\mathrm{C}$-terminus does not interfere with its incorporation into nucleosomes (Sarcinella et al., 2007, Vardabasso et al., 2015). The BirA enzyme added to the C-terminus of H2A.Z is similar in size as GFP, so we do not expect the BirA tag to interfere with H2A.Z-FB's incorporation into chromatin. To confirm this, we harvested micrococcal nuclease-digested chromatin from H2A.Z-FB + Avi-ub-transfected cells and performed mono-nucleosome immunoprecipitation using streptavidin (SA)- or Flag antibody-couple beads (schematically depicted in Fig 2A). As shown in the Coomassie-stained gel (Fig 2B), both the SA- or Flag- immunoprecipitated samples contain stoichiometric amounts of core histones (i.e. roughly equal amounts of H2B, H3 and H4) that co-purify with the immunoprecipitated H2A.Z-FB (lanes 6 and 7 of Fig 2B). Therefore, this indicates that both the non-ubiquitylated and ubiquitylated H2A.Z-FB are assembled into nucleosomes, and that intact nucleosomes are efficiently purified by the Flag- or SA-coupled beads. As an additional control, we also co-expressed the H2A.Z-FB with a K10R mutant form of Avi-ub whereby the lysine within the Avi-tag sequence is mutated to the non-biotinylatable arginine. Mono-nucleosomes harvested from these transfected cells were subjected to immunoprecipitation by SA-beads, and as expected, no detectable amounts of core histones were co-purified. This result confirms that copurification of the core histones (H2B, H3 and H4) with the Avi-ub-H2A.Z-FB is due to their assembly into nucleosomes, and not because of non-specific binding of histones to SA-beads.

We next used Western blot analyses to characterize the SA- or Flagimmunoprecipitated mono-nucleosomes from cells expressing various combinations of H2A.Z-FB and Avi-ub (Fig 2C). By using higher percentage and longer polyacrylamide gels to better resolve the H2A.Z-FB, we were able to 
separate and detect non-ubiquitylated H2A.Z-FB (the fastest migrating band), H2A.Z-FB ubiquitylated with endogenous ubiquitin (the middle band), and those ubiquitylated with Avi-ub (the top band; Fig. 2C). Most importantly, when we normalized the immunoprecipitated nucleosomes based on the amount of $\mathrm{H3}$ pulled down, we observe that the SA-coupled beads specifically immunoprecipitated the slowest migrating Avi-ub-H2A.Z-FB, whereas Flagbeads mostly immunoprecipitated the non-ubiquitylated H2A.Z-FB and a small amount of H2A.Z-FB mono-ubiquitylated with endogenous (non-Avi-tagged) ubiquitin. From cells expressing the H2A.Z-FB K3R3 mutant and Avi-ub, the Flag-IP only pulled down the non-ubiquitylated H2A.Z-FB as expected. Also, from cells expressing WT H2A.Z-FB and the K10R Avi-ub mutant, the SA-beads did not pull down any H2A.Z-FB, nor endogenous histones, demonstrating specificity of the SA-bead immunoprecipitation. Finally, we note that while Avidin-HRP detected both the Avi-ubiqutiylated-H2A.Z-FB and Aviubiquitylated-endogenous H2A.Z/H2A in the input mono-nucleosomes (Fig. 2C, left panel), the SA- immunoprecipitation mainly pulled down the Avi-ub-H2A.Z, and only a small amount of the Avi-ub-endogenous H2A.Z/H2A (Fig. 2C, right panel). Together, these results demonstrate that our experimental system and SA-purification scheme highly enriches for ubiquitylated H2A.Z-containing nucleosomes, thus allowing an in depth study of their biochemical and genomic distribution characteristics.

\section{Ubiquitylated H2A.Z nucleosomes are enriched for transcriptional repressive histone modifications and depleted of activating histone modifications.}

Having established that distinct pools of H2A.Z-FB nucleosomes are pulled down by the SA- and Flag-IPs (depicted in Fig. 2A), we next examined the histone modification patterns on these nucleosomes by Western blot (Fig. 3). We again normalized the samples by $\mathrm{H} 3$ levels in order to compare equivalent amounts of the immunoprecipitated nucleosomes (H3 Western blot panel of Fig. 3). As seen before, the Flag antibody preferentially immunoprecipitated mostly non-ubiquitylated H2A.Z-FB (from the WT-H2A.Z-FB-expressing cells) or only non-ubiquitylated H2A.Z-FB (from the K3R3 mutant-expressing cells) whereas the SA-beads immunoprecipitated the Avi-ubiquitylated form of H2A.Z-FB (Flag 
panel of Fig. 3). Comparison of these two pools of nucleosomes showed similar levels of H3K9me2, H3K9me3 and H4K16ac. However, compared to the nucleosomes that contained mostly non-ubiquitylated H2A.Z-FB, nucleosomes containing Avi-ub-H2A.Z-FB are depleted of H3K4 methylation (most notably for H3K4me2 and H3K4me3), H3K27ac, as well as hyperacetylated H4, all which are general hallmarks for transcriptionally active chromatin. In contrast, the Avi$\mathrm{ub}-\mathrm{H} 2 \mathrm{~A} . \mathrm{Z}$ nucleosomes are enriched for H3K27me3, which is a hallmark for polycomb-silenced facultative heterochromatin. Taken altogether, these characterizations reveal that ubiquitylated H2A.Z-nucleosomes are depleted of histone PTM hallmarks associated with transcriptional activation, but are enriched for H3K27me3 that is associated with the polycomb-silencing pathway.

\section{Genomic analyses of Avi-ub-H2A.Z-FB-containing chromatin.}

The nucleosome IP method we used to purify H2A.Z-FB nucleosomes affords the analysis of the distribution of this modified histone variant on a genome-wide scale. Accordingly, using conditions equivalent to native ChIP, we next extracted DNA from the SA- and Flag-immunoprecipitated nucleosomes (from biological replicate preparations) and performed high-throughput sequencing. In parallel, we performed ChIP-Seq analysis using an antibody against H3 as control for histone density. Overall, we identified $~ 144,000$ peaks in both the SA- and Flag$\mathrm{IP}^{\prime} \mathrm{d}$ samples and found significant $(\sim 60 \%)$ overlap between them. This is expected since the Flag-IP should pull down both non-ubiquitylated and ubiquitylated H2A.Z-FB, whereas the SA-IP specifically enriches the Avi-ubH2A.Z-FB. H2A.Z has been reported to be enriched at the +1 , and to a lesser extent at the -1 , nucleosomes flanking transcription start sites (TSSs) of many genes. When the SA- and Flag-IP peaks were mapped to the gene borders (transcription start sites, TSSs, and transcription termination sites, TTSs) we indeed observe a strong peak at +1 nucleosome position and a weaker peak at the -1 nucleosome position for both SA- and Flag-IPs (Fig. 4A), indicating that H2A.Z-FB is correctly targeted to endogenous H2A.Z sites within the genome. Interestingly, when the SA- and Flag-IP peaks were separately mapped to genes with no-, low-, or high-expression (based on analysis of polyA+ RNA-seq data from untransfected $293 \mathrm{~T}$ cells), there is a comparable degree of enrichment of SA- 
IP peaks at the TSSs of these three groups of genes, whereas there are significantly higher Flag-IP peaks at low- or high-expression genes compared to non-expressed genes (Fig. 4B). This difference is apparent as a 'double-dip' around the TSS when the SA-IP signal is normalized to the Flag-IP signal (3rd panel of Fig. 4B). These findings suggest that while roughly equal amounts of Avi-ub-H2A.Z-FB are found at the +1 nucleosome position of genes irrespective of their expression status, significantly more total H2A.Z-FB (most likely corresponding to non-ubiquitylated H2A.Z-FB) is enriched at the +1 and -1 nucleosome positions of active compared to inactive genes. It is of interest to note that the Flag-IP peaks flanking the TSSs are almost identical for both low- and high-expressing genes, suggesting that while active promoters in general have higher amounts of H2A.Z compared to non-active promoters, the amount of H2A.Z at the $+1 /-1$ nucleosomes does not correlate with gene expression levels (low vs. high expression). Lastly, we also note that higher amounts of both SAand Flag-IP'd H2A.Z-FB were found at the $3^{\prime}$ end of non-expressing genes compared to low- or high-expression genes (Fig 4B), which is consistent with previous H2A.Z ChIP-microarray studies in yeast that showed accumulation of H2A.Z at gene bodies of transcriptionally inactive genes (Hardy et al., 2009).

To further analyze the nucleosome IP-seq data, we first merged all peaks detected in the SA- or Flag-IPs, resulting in 198,000 peaks, and determined the relative enrichment or depletion of the SA-IP signal at each peak by comparing the read density of the SA-IP to the Flag-IP using statistical tests. Peaks where the SA-IP signal is significantly enriched are identified as "Avi-enriched" (12,403 peaks), whereas those with significantly depleted SA-IP signal are identified as "Avi-depleted" (18,881 peaks). Lastly, peaks that do not reach statistical significance for either enrichment or depletion are classified as "no-enrichment". These three categories essentially correspond to IP-seq peaks enriched for Aviubiquitylated H2A.Z-FB, non-ubiquitylated H2A.Z-FB, or a roughly equal mix of both. By examining the Avi-enriched vs. Avi-depleted peaks at identified gene promoters and stratifying them based on expression levels, we found that $>70 \%$ of the Avi-enriched promoters are transcriptionally silent (defined by $<5$ RPKM based on RNA-seq data) whereas $<30 \%$ of Avi-depleted promoters are 
transcriptionally silent (Fig. 4C; odds ratio = 7.2, p-value $<2.2 \mathrm{e}-16$, Fisher's exact test). The distribution of no enrichment peaks across promoters of different expression levels is in between that seen for Avi-enriched and Avi-depleted peaks, which likely reflects the mixture of both ubiquitylated and nonubiquitylated H2A.Z at these promoters. Lastly, comparison of the Avi-enriched vs. Avi-depleted peaks at gene bodies also showed a higher fraction of Avienriched genes at inactive genes; however, the difference is not as striking as seen at promoters (odds ratio $=3.2, \mathrm{p}$-value $<2.2 \mathrm{e}-16$, Fisher's exact test). Altogether, these analyses show that ubiquitylated and non-ubiquitylated H2A.Z are respectively enriched on transcriptionally inactive and transcriptionally active promoters, which mirrors the distinct histone PTMs associated with transcriptional repression and activation found on the respective Avi-ub- and non-ubiquitylated-H2A.Z-FB nucleosomes (Fig. 3).

To further characterize the Avi-enriched vs. Avi-depleted promoters, we examined their expression profiles across 55 different tissues / cell types, and expressed the data as the fraction of Avi-enriched or Avi-depleted promoters/genes that is 'over-expressed' (i.e. expression $>$ average expression) in different tissues or cell types. As shown in Fig. 4D, we found that Avienriched or Avi-depleted promoters have distinct expression profiles across different tissues. For example, Avi-enriched promoters are not frequently overexpressed in the tissues/cell types tested whereas Avi-depleted promoters (i.e. enriched for non-ubiquitylated H2A.Z) are over-expressed in higher percentages of tissues / cell types tested. These profiles suggest that Avi-enriched genes have a more restricted expression pattern in different cell types, which is consistent with a negative regulatory role of $\mathrm{H} 2 \mathrm{~A} . \mathrm{Z}$ ubiquitylation on gene expression.

Lastly, we compared the distribution of Avi-enriched vs. Avi-depleted peaks across different genomic regions such as promoters, enhancers, exons, introns and intergenic regions. More specifically, we examined the percentage occupancy (i.e. coverage) of these genomic elements by the Avi-enriched and Avi-depleted peaks to determine the relative densities of these peaks within each of the elements (Fig. 4E). Interestingly, Avi-depleted peaks have the highest 
percentage coverage at promoters and next at enhancers, whereas Avi-enriched peaks have the highest percentage coverage in exons, then at promoters, but least of all at enhancers. The higher occupancy of H2A.Zub (compared to nonubiquitylated H2A.Z) at exons is intriguing and suggests that it may also have a a function outside of transcription initiation. The data also found significant occupancy of non-ubiquitylated H2A.Z at predicted active enhancers, but an apparent depletion of $\mathrm{H} 2 \mathrm{~A} . \mathrm{Zub}$ at these elements. Insofar as active enhancers are defined by high levels of H3K4me1 and H3K27ac, this observation is consistent with, and possibly explained by, our biochemical analyses showing that H2A.Zub nucleosomes have low levels of these modifications whereas nonubiquitylated H2A.Z nucleosomes have high levels of these modifications (Fig 3).

\section{Validation and characterization of Avi-enriched and Avi-depleted promoters by gene-specific ChIP.}

Having identified distinct sets of Avi-enriched and Avi-depleted genes, we chose a number of representative promoters (12 promoters from each group) for validation by gene-specific ChIP. We first performed ChIPs using SA- or Flagcoupled beads, and used qPCR with primers corresponding to regions flanking the TSSs of the 24 tested genes for quantitative analyses. We normalized the SAIP signals to the Flag-IP signals and plotted the individual gene data as shown in Fig 5A, and we combined the ChIP data of the two groups of genes in a strip plot comparison with statistical analyses (Fig. 5B). In addition, we examined the H3K4me3, H3K27me3, and total H2A.Z levels at the same promoters by ChIP, and normalized the data to the total H3 ChIP signal (normalizing for nucleosome density) (Figs. 5A and B). By combining the ChIP data from the individual genes in the Avi-enriched or Avi-depleted promoters, we were able to see statistically relevant trends more clearly (Fig. 5B). First, as expected, the Avi-signal (SA-IP normalized to Flag-IP signal) is significantly higher for the Avi-enriched group compared to the Avi-depleted group, indicating an enrichment of Avi-ubH2A.Z-FB at those promoters ( $p$ value $<0.0005$, Wilcoxon signed-rank test; Fig. 5B). In contrast, there was no evidence for differential occupancy of total H2A.Z at the promoters of both groups of genes, suggesting similar amounts of total H2A.Z at these promoters. Also, consistent with the histone PTM trends 
observed by our nucleosome-IP-Western blots, the Avi-enriched promoters are hyper-methylated for H3K27 but hypo-methylated for H3K4, whereas the reverse trend is seen at the Avi-depleted promoters ( $p$ value $<0.017$ for H3K27me3 ChIP, and p value $<0.0015$ for H3K4me3 ChIP; Wilcoxon signedranked test). From these data, the most striking feature of the Avi-enriched promoters is the consistent hypo-methylation of H3K4. This observation is not only consistent with our previous biochemical analysis (Fig. 3) and the connections between H2A.Z ubiquitylation and transcriptional silencing, but further suggests a possible incompatibility or mechanistic antagonism between H2A.Z ubiquitylation and H3K4 methylation.

\section{Identification of $\mathbf{H 2 A . Z u b - n u c l e o s o m e ~ c o - p u r i f y i n g ~ p r o t e i n s . ~}$}

We next asked whether there are also chromatin binding proteins that differentially associate with ubiquitylated- and non-ubiquitylated H2A.Znucleosomes. Accordingly, we co-transfected various combinations of WT or mutant H2A.Z-FB and Avi-ub, harvested mono-nucleosomes from those cells, and performed nucleosome immunoprecipitation using SA- vs. Flag-coupled beads. We again normalized the amount of nucleosomes immunoprecipitated and loaded per lane by H3 density and blotted for various chromatin interacting proteins to examine their binding patterns (Fig. 6A). We previously identified Brd2 as an H2A.Z-nucleosome binding protein and found that it is recruited to androgen receptor-regulated genes upon hormone induction in an H2A.Zdependent manner (Draker et al., 2012). Consistent with Brd2 having a role in transcriptional activation, we found that it only co-purifies with the Flagimmunoprecipitated (i.e. H2A.Z-FB-containing) nucleosomes, but not with the SA- immunoprecipitated (i.e. Avi-ub-H2A.Z-FB-containing) nucleosomes. In contrast, menin, a component of the MLL (H3K4 methyltransferase) complex, is found at similar levels in both SA- and Flag-IPs, with a slight increase in level in the SA-IP sample. Given the apparent anti-correlation between H3K4 methylation and H2A.Z ubiquitylation, we next tested whether LSD1, an H3K4me2 de-methylase, co-purifies with the Avi-ub-H2A.Z-FB nucleosomes. Strikingly, both LSD1 and DNMT3L, an interacting partner of the de novo DNA methyltransferases DNMT3a/b, preferentially co-purified with Avi-ub-H2A.Z- 
FB nucleosomes. The preferential enrichment and recruitment of these proteins may facilitate H3K4 de-methylation and DNA methylation of H2A.Zubnucleosomes and contribute to transcriptional silencing of H2A.Zub-targeted genes.

Lastly, given that our genomic analyses suggested that H2A.Zub is depleted at enhancer elements, we asked whether enhancer-associated proteins differentially associate with ubiquitylated vs. non-ubiquitylated H2A.Z. To this end, recent studies have found that the cohesin complex and CTCF may have roles in mediating enhancer-promoter interactions and; therefore, we tested whether the cohesin components Rad21 and SMC1 or CTCF differentially associate with the ubiquitylated / non-ubiquitylated H2AZ-FB-containing nucleosomes. Given that cohesin and CTCF are reported to facilitate enhancer-promoter interactions, we initially hypothesized that these factors may preferentially associate with nucleosomes containing the non-ubiquitylated form of H2A.Z-FB. However, to our surprise, we found that Rad21, SMC1, and CTCF were all preferentially recovered in the SA- immunoprecipitates, but minimally in the Flagimmunoprecipitates (Fig. 6A). This suggests that these chromatin binding proteins preferentially associate with nucleosomes containing the ubiquitylated form of H2A.Z. To confirm that the co-purification was dependent on ubiquitylated H2A.Z, we performed SA-immunoprecipitation using nucleosomes harvested from cells transfected with the non-ubiquitylatable K3R3 H2A.Z-FB and Avi-ub. As shown in Fig. 6B, significantly less SMC1 and CTCF were recovered in the SA- immunoprecipitates when the H2A.Z ubiquitylation sites were mutated on the H2A.Z-FB. The residual amounts of SMC1 and CTCF immunoprecipitated with the K3R3 H2A.Z-FB nucleosomes are likely due to their interaction with the biotinylated endogenous $\mathrm{H} 2 \mathrm{~A} . \mathrm{Zub}$ (the lower band detected in the Avidin-HRP). This result confirms that cohesin components and CTCF preferentially interact with Avi-ub-H2A.Z-FB nucleosomes. Although the functional significance of these interactions are not yet fully understood, these findings raise intriguing functional and mechanistic possibilities related to H2A.Z ubiquitylation. 


\section{Discussion}

In this study, we developed a unique method to specifically purify H2A.Zub nucleosomes for biochemical and genomics analyses. Western blot analyses revealed distinct patterns of histone PTMs that co-exist with H2A.Zub or nonubiquitylated H2A.Z in the context of the nucleosome, and these PTMs on the respective nucleosomes are either all associated with transcriptional repression or activation. These findings support the general hypothesis that combinatorial histone PTMs work in concert to mediate downstream effects ( $\mathrm{Ng}$ and Cheung, 2016), and the possibility of PTM cross-talks that shapes the overall histone PTMs that co-function with H2A.Zub. For example, our previous work showed that H2A.Z ubiquitylation is mediated by the RING1b E3 ligase of the PRC1 complex (Sarcinella et al., 2007) and; therefore, our observed coupling of H2A.Z ubiquitylation and H3K27 methylation is consistent with the known interplay between PRC2-meditated H3K27 methylation and PRC1 recruitment (Aranda et al., 2015). Work by other labs also showed the existence of an antagonistic switch between H3K27 methylation and acetylation such that loss of H3K27 methylation can lead to increases in the H3K27ac levels (Pasini et al., 2010). Therefore, the reciprocal patterns of $\mathrm{H} 3 \mathrm{~K} 27$ methylation and acetylation on ubiquitylated and non-ubiquitylated H2A.Z nucleosomes are consistent with this known reciprocal relationship between $\mathrm{H} 3 \mathrm{~K} 27$ methylation and acetylation, and also raise the possibility of functional coupling of H2A.Z de-ubiquitylation with H3K27 acetylation.

Our ChIP-seq analyses also provided the first genome-wide view of the distribution and enrichment of $\mathrm{H} 2 \mathrm{~A}$.Zub within the human genome. Insofar as only a fraction of total H2A.Z in 293T cells is mono-ubiquitylated, we initially considered that this subpopulation of $\mathrm{H} 2 \mathrm{~A} . \mathrm{Z}$ is clustered and restricted to a subset of all genes associated with H2A.Z. However, our data revealed that this is not the case. Instead, ubiquitylated and non-ubiquitylated H2A.Z are extensively intermingled throughout the genome. We further observed that $\mathrm{H} 2 \mathrm{~A}$.Zub is found at similar levels at the TSSs of both transcriptionally active and inactive genes, whereas total $\mathrm{H} 2 \mathrm{~A} . \mathrm{Z}$ is significantly more abundant at the 
promoters of transcriptionally active genes. This suggests that, on average, active and inactive genes do not differ significantly in the absolute amount of H2A.Zub flanking their TSSs, but active gene promoters have higher levels of total (presumably mostly non-ubiquitylated) H2A.Z. The enrichment of nonubiquitylated H2A.Z at active genes fits well with the known general association between H2A.Z and transcriptional activity in human cells (Giaimo et al., 2019, Subramanian et al., 2015). Furthermore, we also note that the similar levels of H2A.Z were found at the TSSs of both low- and high-expressing genes, which is consistent with the model that $\mathrm{H} 2 \mathrm{~A}$. $\mathrm{Z}$ functions to poise genes for transcriptional activation, but may not directly affect the rate of transcription (Larochelle and Gaudreau, 2003). Altogether, our ChIP-seq data suggest that the transcriptional activity of genes does not correlate with the absolute presence or absence of either non-ubiquitylated or ubiquitylated H2A.Z, but is instead determined by the relative ratios of these different forms of $\mathrm{H} 2 \mathrm{~A} . \mathrm{Z}$ at promoters.

In support of this idea, our identification of significant Avi-enriched $(\approx \mathrm{H} 2 \mathrm{~A} . \mathrm{Zub}-$

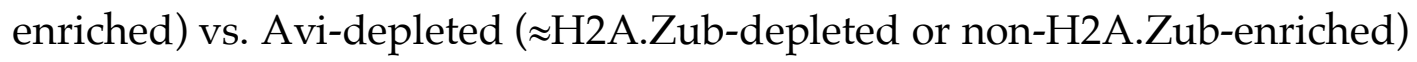
ChIP-Seq peaks revealed clear correlations between promoter enrichment of $\mathrm{H} 2 \mathrm{~A} . \mathrm{Zub}$ with gene-silencing, and promoter enrichment of non-ubiquitylated H2A.Z with gene activation. Gene-specific ChIP assays further verified these correlations, and more interestingly, we found that the H2A.Zub-enriched promoters are not only hypermethylated at H3K27me3, but also hypomethylated at H3K4me3, whereas the reverse trend is seen for the H2A.Zubdepleted promoters. These trends exactly mirror the patterns of histone modification identified from our Western blot analyses of purified H2A.Zubnucleosomes. Therefore, our study provides corroborating biochemical and genomics data indicating reciprocal correlations between H2A.Z ubiquitylation and H3K4- / H3K27-methylation levels. Previous ChIP-seq analyses of H2A.Z localization in mouse and human ES cells have identified a strong correlation/ co-enrichment of H2A.Z and H3K4me3, especially at promoters and enhancers (Ku et al., 2012, Hu et al., 2013). Moreover, knockdown of H2A.Z or MLL4 in mouse ES cells has been reported to respectively cause decreased 
H3K4me3 or H2A.Z levels at some enhancers, suggesting that deposition of these epigenetic marks are functionally linked in some circumstances (Hu et al., 2013). From our study, we now find an anti-correlation between H2A.Zub and $\mathrm{H} 3 \mathrm{~K} 4 \mathrm{me} 3$ and this thus raises the possibility that ubiquitylation of $\mathrm{H} 2 \mathrm{~A} . \mathrm{Z}$ antagonizes or reverses the connection between H2A.Z and H3K4 methylation. Indeed, our discovery that the H3K4 de-methylase LSD1 preferentially associates with H2A.Zub nucleosomes supports this possibility and further suggests potential crosstalk between these modifications (further discussed below).

Comparison of the genome-wide distribution of H2A.Zub-enriched and depleted peaks at different functional domains also showed striking differences in their occupancies at promoters, enhancers and exons. Consistent with the known enrichment of H2A.Z at promoters and enhancers ( $\mathrm{Ku}$ et al., 2012, Hu et al., 2013), H2A.Zub-depleted (i.e. mostly non-ubiquitylated H2A.Z) peaks proportionally occupy more territories at promoters and next at enhancers, compared to H2A.Zub-enriched peaks. In contrast, H2A.Zub-enriched peaks occupy larger areas at exons, suggesting that H2A.Z ubiquitylation may have additional non-transcription initiation-related functions at exons. The greatest difference between H2A.Zub-enriched and -depleted peaks are their relative occupancies at active enhancer elements. In this analysis, active enhancers are defined by co-enrichment of H3K4me1 and H3K27ac, so the low occupancy of H2A.Zub at enhancers complements our observed depletion of H3K4me1/2/3 and H3K27ac on H2A.Zub nucleosomes by Western blot analyses. H2A.Z has recently been shown to be important for transcription of enhancer RNAs (eRNAs) and for enhancer functions (Valdes-Mora et al., 2017), so our data suggest that it is the non-ubiquitylated form of H2A.Z that facilitates eRNA transcription and enhancer functions. Moreover, given the connections between H2A.Z ubiquitylation and transcriptional silencing, our data also raise the possibility that H2A.Zub may be actively excluded or is functionally antagonistic to eRNA transcription and enhancer activities.

We and others have previously found that H2A.Z-containing nucleosomes bind a number of chromatin binding proteins that mediate downstream functions 
(Draker et al., 2012, Kim et al., 2013, Vardabasso et al., 2015). For example, we found that the transcriptional regulator Brd2 binds H2A.Z nucleosomes through combinatorial interactions with H2A.Z and acetylated H4 (Draker et al., 2012). More recently, Hake and colleagues also found that PWWP2A binds H2A.Z nucleosomes through multivalent interactions (Punzeler et al., 2017). In the current study, we found that Brd2 co-purifies with non-ubiquitylated but not with ubiquitylated H2A.Z nucleosomes, which fits the known connections between Brd2 and transcriptional activation, and also complements a previous report that suggested H2A.Z ubiquitylation antagonizes Brd2 binding in ES cells (Surface et al., 2016). In contrast to Brd2, we found several transcription silencing factors, such as LSD1 and DNMT3L, that preferentially interact with ubiquitylated H2A.Z nucleosomes. LSD1, also known as KDM1A, was the first histone de-methylase identified that targets $\mathrm{H} 3 \mathrm{~K} 4 \mathrm{me} 2 / \mathrm{me} 1$, and functions as a transcriptional co-repressor in specific contexts (Shi et al., 2004). Therefore, physical recruitment of LSD1 or other H3K4 de-methylases to ubiquitylated H2A.Z nucleosomes may be a direct mechanism leading to the striking anticorrelation between H3K4 methylation and H2A.Z ubiquitylation seen in our biochemical and ChIP analyses. LSD1 is part of two complexes, CoREST and NuRD (Shi et al., 2005, Wang et al., 2009b), that both contain histone deacetylases, so the low levels of both $\mathrm{H} 3 \mathrm{~K} 4$ methylation and $\mathrm{H} 4$ acetylation on $\mathrm{H} 2 \mathrm{~A}$.Zub nucleosomes could also reflect recruitment of multiple histone modifying activities through these complexes. Finally, LSD1 has also been shown to interact with DNMT1 and DNMT3B, and is important for maintaining global DNA methylation (Wang et al., 2009a, Brenner et al., 2016). The co-purification of LSD1 and DNMT3L with H2A.Zub nucleosomes suggests that these nucleosomes may also have high levels of DNA methylation. Our original characterization of $\mathrm{H} 2 \mathrm{~A}$.Zub found that it was enriched on the inactive $\mathrm{X}$ chromosomes in female mammalian cells (Sarcinella et al., 2007), which are stably silenced by multiple epigenetic mechanisms including H3K27 methylation, loss of $\mathrm{H} 3 \mathrm{~K} 4$ methylation, $\mathrm{H} 3$ / H4 de-acetylation, $\mathrm{H} 2 \mathrm{~A}$ ubiquitylation and DNA methylation (Gendrel and Heard, 2014). Therefore, the chromatin proteins we found co-purifying with H2A.Zub are consistent with the 
convergence of multiple histone/chromatin-modifying mechanisms on H2A.Zcontaining chromatin to mediate stable transcriptional silencing.

Our nucleosome-binding assay also uncovered that CTCF and cohesin components preferentially associate with ubiquitylated H2A.Z nucleosomes. Both CTCF and the cohesin complex are chromosomal architectural proteins that mediate long-range chromatin/chromosome interactions, and they are also important for establishing the boundaries of Topologically Associating Domains (TADs) that define the functional domains within the genome where permissive and high frequencies of chromatin interactions occur (Song and Kim, 2017). Therefore, the co-purification of these proteins raises the possibility that ubiquitylated H2A.Z's function may also involve higher order chromatin folding/topology. Earlier ChIP-seq studies have found enrichment of H2A.Z at CTCF and Rad21 (a cohesin subunit) binding sites within the human genome (Barski et al., 2007, Lashgari et al., 2017) and; therefore, our nucleosome-IP results provide direct physical interaction data to confirm and support the previous correlative findings. At present, the functional significance of these interactions is not known. CTCF and cohesin perform multiple cellular functions, including enhancer-blocking / insulator functions for CTCF, sister chromatid cohesion for cohesin, and they are both involved in transcriptional regulation as well (for review, see Merkenschlager and Nora, 2016). Given their interactions are specific to ubiquitylated H2A.Z, it is possible that these proteins act together in the context of transcriptional silencing, either directly at the gene level, or through the assembly or maintenance of repressive domains within the genome. CTCF was first identified as a DNA binding protein that binds and represses transcription of the chicken c-myc gene (Lobanenkov et al., 1990). CTCF has also been found to recruit HDACs to silence transcription in transfection-based reporter assays (Lutz et al., 2000), so it is possible that CTCF engages multiple histone modifying mechanisms to silence target genes. Cohesin is often associated with active enhancers and promoters, but there are also connections with gene silencing (Merkenschlager and Odom, 2013). Early work in S. cerevisae showed that cohesin is targeted to transcriptionally silenced chromatin (Chang et al., 2005). In multi-cellular eukaryotes, cohesin directly interacts with the PRC1 
complex and has been proposed to target Polycomb-group proteins to active genes (Strubbe et al., 2011, Dorsett and Kassis, 2014). Therefore, cohesin may physically associate with ubiquitylated H2A.Z through its direct interaction with PRC1. The transcriptional regulatory functions of CTCF / cohesin are complex, involving multiple levels of direct and indirect mechanisms. Therefore, our discovery of their physical association with H2A.Z ubiquitylation raises new questions about their functional links to chromatin and nucleosomes.

\section{Acknowledgements}

We thank Keyur Advaryu and Kashif Aziz Khan for scientific discussions and general support, and Emanuel Rosonina for critical reading of the manuscript. MKN was funded by a Canadian NSERC Postgraduate Scholarship, and this work was funded by in part by operating grants from the Canadian Cancer Society Research Institute and the Canadian Institutes of Health Research awarded to PC, as well as a CIHR Foundation grant awarded to BJB.

\section{Author contributions}

PC conceived the method and experimental designs, and MKN performed all the experimental work. UB performed all the ChIP-sequencing data analyses. PC wrote the manuscript with contributions from all co-authors.

\section{Declaration of Interests}

The authors declare no competing interests. 


\section{Figure Legends}

Figure 1. Validation of the auto-biotinylation of Avi-ubiquitylated H2A.ZFlag-BirA. (A) Cartoon diagram illustrating the H2A.Z-Flag-BirA (H2A.Z-FB) fusion and its auto-biotinylation upon ubiquitylation with Avi-tagged ubiquitin (Avi-Ub). (B) $293 \mathrm{~T}$ cells were co-transfected with wild type (WT) or nonubiquitylatable (K3R3) H2A.Z-FB with or without Avi-Ub. Whole cell or nuclear extracts were harvested from the transfected cells and total H2A.Z-FB or biotinylated H2A.Z-FB were detected by Western blots using Flag antibody or horse radish peroxidase-couple avidin (Avidin-HRP). H3 was used as loading control. (C) Mono-nucleosomes were prepared from 293T cells transfected with the indicated NLS-Flag-BirA, H2A.Z-FB (WT or non-ubiquitylatable KR mutant) and Avi-Ub combinations. The presence of Flag tagged and biotinylated proteins were detected by Western blots using Flag antibody or Avidin-HRP. The molecular weight (MW) markers show the relative sizes of the bands detected by the Western blots.

\section{Figure 2. Separation of distinct pools of H2A.Z-FB nucleosomes by} streptavidin (SA) or Flag antibody-immunoprecipitation. (A) Cartoon diagram showing the expected types of H2A.Z-FB nucleosomes found in the H2A.Z-FB + Avi-Ub transfected cells and the distinct types of nucleosomes pulled down by SA or Flag antibody immunoprecipitation. (B) Coomassie stained gel of the input mono-nucleosome fraction and SA- or Flag antibody-immunoprecipitated nucleosomes. * indicates non-specific bands often seen in SA-IPs (C) Western blot analysis of the input and immunoprecipitated nucleosomes using Flag antibody and Avidin-HRP. The different types of ubiquitylated and non-ubiquitylated H2A.Z-FB (illustrated on the right hand side and marked by single, double and triple asterisks) were resolved on long SDS polyacrylamide gels and detected by Flag antibody.

Figure 3. Distinct patterns of histone modifications are found on the Aviubiquitylated or mostly non-ubiquitylated H2A.Z-FB nucleosomes. 293T cells were transfected with various H2A.Z-FB and Avi-Ub constructs as shown, and 
the levels of different histone modifications on the SA- or Flag antibodyimmunoprecipitated nucleosomes were examined by Western blotting. Equal amounts of the purified nucleosomes were loaded on each lane as indicated by the comparable H3 levels in each lane. The K10R Avi-Ub is the nonbiotinylatable Avi-Ub control to show the biotinylation-dependent pulldown of nucleosomes by SA-immunoprecipitation.

Figure 4. Genome-wide analysis of the ubiquitylated H2A.Z- or total H2A.Zcontaining nucleosomes. Total genomic DNA extracted from the SA- or Flag antibody-immunoprecipitated nucleosomes were sent for Illumina sequencing and analyzed by bioinformatics tools. (A) Total SA- or Flag- antibody IP peaks were compiled and mapped relative to transcription start sites (TSSs) and transcription termination sites (TTSs) of all genes. Number of aligned genes at each position are indicated at the bottom. (B) SA- and Flag antibody-IP peaks were mapped relative to the TSSs and TTSs of no-expression (grey line), lowexpression (red line) or high-expression (yellow line) genes. (C) SA- and Flag antibody-IP peaks were compared relative to one another and statistically determined as either Avi-enriched, Avi-depleted, or no enrichment. These different categories of peaks were mapped to promoters and gene bodies and then stratified based on the RNA-seq determined expression levels of the genes. (D) Expression levels of Avi-enriched (orange), Avi-depleted (red) or no enrichment promoters were analyzed across 55 different tissue or cell types. The fraction of tissues/cells that show greater than the average expression of each gene were plotted and color coded based on Avi-enrichment, -depletion or no enrichment. (E) Avi-enriched or Avi-depleted peaks were categorized according to ENCODE-defined promoters, enhancers, exons, introns, and intergenic regions. The real estate in each category occupied by Avi-enriched or Avidepleted peaks were expressed as percent coverage (the percent of basepairs occupied by the respective peak types).

Figure 5. Gene-specific ChIP analysis of representative Avi-enriched and Avidepleted promoters. (A) 12 representative gene promoters from the ChIP-seq identified list of Avi-enriched or Avi-depleted promoters were analyzed by 
ChIP-qPCR assays. The SA-ChIP signal for each gene was normalized to the Flag-ChIP signal, whereas the H2A.Z, H3K27me3 and H3K4me3 ChIP signals were normalized to the total H3 ChIP signal to normalize for the nucleosome densities at the tested promoters. (B) The ChIP data from the individual groups of 12 genes were combined and their relative enrichment plotted. The median of each grouping was calculated and marked for statistical comparisons.

\section{Figure 6. Differential binding of different chromatin-binding proteins to either ubiquitylated or mostly non-ubiquitylated H2A.Z-containing}

nucleosomes. (A) Mono-nucleosomes from 293T cells expressing different types of H2A.Z-FB and Avi-Ub were harvested and immunoprecipitated with SA- of Flag antibody-coupled beads. The relative amounts of different chromatin binding proteins co-purifying with normalized amounts of the SA- or Flag antibody-purified nucleosomes were determined by Western blot analyses. (B) Mono-nucleosomes from 293T cells expressing either WT or non-ubiquitylatable (K3R3) H2A.Z-FB and Avi-Ub were immunoprecipitated with either SA- or Flag antibody beads and the relative amounts of SMC1 and CTCF co-purifying with the different immunoprecipitated nucleosomes were determined by Western blotting. 


\section{References}

Adam, M., Robert, F., Larochelle, M., and Gaudreau, L. (2001). H2A.Z is required for global chromatin integrity and for recruitment of RNA polymerase II under specific conditions. Molecular and cellular biology 21, 6270-6279.

Aranda, S., Mas, G., and Di Croce, L. (2015). Regulation of gene transcription by Polycomb proteins. Sci Adv 1, e1500737.

Barski, A., Cuddapah, S., Cui, K., Roh, T.Y., Schones, D.E., Wang, Z., Wei, G., Chepelev, I., and Zhao, K. (2007). High-resolution profiling of histone methylations in the human genome. Cell 129, 823-837.

Bonev, B., and Cavalli, G. (2016). Organization and function of the 3D genome. Nat Rev Genet 17, 661-678.

Brenner, C., Luciani, J., Bizet, M., Ndlovu, M., Josseaux, E., Dedeurwaerder, S., Calonne, E., Putmans, P., Cartron, P.F., Defrance, M., et al. (2016). The interplay between the lysine demethylase KDM1A and DNA methyltransferases in cancer cells is cell cycle dependent. Oncotarget 7, 58939-58952.

Buschbeck, M., and Hake, S.B. (2017). Variants of core histones and their roles in cell fate decisions, development and cancer. Nat Rev Mol Cell Biol 18, 299-314.

Chang, C.R., Wu, C.S., Hom, Y., and Gartenberg, M.R. (2005). Targeting of cohesin by transcriptionally silent chromatin. Genes Dev 19, 3031-3042.

Cheung, P., Allis, C.D., and Sassone-Corsi, P. (2000). Signaling to chromatin through histone modifications. Cell 103, 263-271.

Dorsett, D., and Kassis, J.A. (2014). Checks and balances between cohesin and polycomb in gene silencing and transcription. Curr Biol 24, R535-539.

Draker, R., and Cheung, P. (2009). Transcriptional and epigenetic functions of histone variant H2A.Z. Biochem Cell Biol 87, 19-25.

Draker, R., Ng, M.K., Sarcinella, E., Ignatchenko, V., Kislinger, T., and Cheung, P. (2012). A combination of H2A.Z and H4 acetylation recruits Brd2 to chromatin during transcriptional activation. PLoS genetics 8, e1003047.

Draker, R., Sarcinella, E., and Cheung, P. (2011). USP10 de-ubiquitylates the histone variant H2A.Z and both are required for androgen receptor-mediated gene activation. Nucleic Acids Res 39, 3529-3542.

Gendrel, A.V., and Heard, E. (2014). Noncoding RNAs and epigenetic mechanisms during X-chromosome inactivation. Annu Rev Cell Dev Biol 30, 561580 . 
Giaimo, B.D., Ferrante, F., Herchenrother, A., Hake, S.B., and Borggrefe, T. (2019). The histone variant H2A.Z in gene regulation. Epigenetics Chromatin 12, 37.

Giaimo, B.D., Ferrante, F., Vallejo, D.M., Hein, K., Gutierrez-Perez, I., Nist, A., Stiewe, T., Mittler, G., Herold, S., Zimmermann, T., et al. (2018). Histone variant H2A.Z deposition and acetylation directs the canonical Notch signaling response. Nucleic Acids Res 46, 8197-8215.

Hardy, S., Jacques, P.E., Gevry, N., Forest, A., Fortin, M.E., Laflamme, L., Gaudreau, L., and Robert, F. (2009). The euchromatic and heterochromatic landscapes are shaped by antagonizing effects of transcription on H2A.Z deposition. PLoS Genet 5, e1000687.

Hu, G., Cui, K., Northrup, D., Liu, C., Wang, C., Tang, Q., Ge, K., Levens, D., Crane-Robinson, C., and Zhao, K. (2013). H2A.Z facilitates access of active and repressive complexes to chromatin in embryonic stem cell self-renewal and differentiation. Cell Stem Cell 12, 180-192.

Jenuwein, T., and Allis, C.D. (2001). Translating the histone code. Science 293, 1074-1080.

Keogh, M.C., Mennella, T.A., Sawa, C., Berthelet, S., Krogan, N.J., Wolek, A., Podolny, V., Carpenter, L.R., Greenblatt, J.F., Baetz, K., et al. (2006). The Saccharomyces cerevisiae histone H2A variant Htz1 is acetylated by NuA4. Genes Dev 20, 660-665.

Kim, K., Punj, V., Choi, J., Heo, K., Kim, J.M., Laird, P.W., and An, W. (2013). Gene dysregulation by histone variant H2A.Z in bladder cancer. Epigenetics Chromatin 6, 34.

Kobor, M.S., Venkatasubrahmanyam, S., Meneghini, M.D., Gin, J.W., Jennings, J.L., Link, A.J., Madhani, H.D., and Rine, J. (2004). A protein complex containing the conserved Swi2/Snf2-related ATPase Swr1p deposits histone variant H2A.Z into euchromatin. PLoS Biol 2, E131.

Kornberg, R.D. (1974). Chromatin structure: a repeating unit of histones and DNA. Science 184, 868-871.

Kouzarides, T. (2007). Chromatin modifications and their function. Cell 128, 693705.

Ku, M., Jaffe, J.D., Koche, R.P., Rheinbay, E., Endoh, M., Koseki, H., Carr, S.A., and Bernstein, B.E. (2012). H2A.Z landscapes and dual modifications in pluripotent and multipotent stem cells underlie complex genome regulatory functions. Genome Biol 13, R85.

Kusch, T., Florens, L., Macdonald, W.H., Swanson, S.K., Glaser, R.L., Yates, J.R., 3rd, Abmayr, S.M., Washburn, M.P., and Workman, J.L. (2004). Acetylation by 
Tip60 is required for selective histone variant exchange at DNA lesions. Science 306, 2084-2087.

Larochelle, M., and Gaudreau, L. (2003). H2A.Z has a function reminiscent of an activator required for preferential binding to intergenic DNA. Embo J 22, 45124522.

Lashgari, A., Millau, J.F., Jacques, P.E., and Gaudreau, L. (2017). Global inhibition of transcription causes an increase in histone H2A.Z incorporation within gene bodies. Nucleic Acids Res 45, 12715-12722.

Lau, P.N.I., and Cheung, P. (2013). Elucidating combinatorial histone modifications and crosstalks by coupling histone-modifying enzyme withi biotin ligase activity. Nucleic Acids Res 41, e49.

Law, C., and Cheung, P. (2015). Expression of Non-acetylatable H2A.Z in Myoblast Cells Blocks Myoblast Differentiation through Disruption of MyoD Expression. The Journal of biological chemistry 290, 13234-13249.

Lobanenkov, V.V., Nicolas, R.H., Adler, V.V., Paterson, H., Klenova, E.M., Polotskaja, A.V., and Goodwin, G.H. (1990). A novel sequence-specific DNA binding protein which interacts with three regularly spaced direct repeats of the CCCTC-motif in the 5'-flanking sequence of the chicken c-myc gene. Oncogene 5, 1743-1753.

Lutz, M., Burke, L.J., Barreto, G., Goeman, F., Greb, H., Arnold, R., Schultheiss, H., Brehm, A., Kouzarides, T., Lobanenkov, V., et al. (2000). Transcriptional repression by the insulator protein CTCF involves histone deacetylases. Nucleic Acids Res 28, 1707-1713.

Meneghini, M.D., Wu, M., and Madhani, H.D. (2003). Conserved histone variant H2A.Z protects euchromatin from the ectopic spread of silent heterochromatin. Cell 112, 725-736.

Merkenschlager, M., and Nora, E.P. (2016). CTCF and Cohesin in Genome Folding and Transcriptional Gene Regulation. Annu Rev Genomics Hum Genet 17, 17-43.

Merkenschlager, M., and Odom, D.T. (2013). CTCF and cohesin: linking gene regulatory elements with their targets. Cell 152, 1285-1297.

Millar, C.B., Xu, F., Zhang, K., and Grunstein, M. (2006). Acetylation of H2AZ Lys 14 is associated with genome-wide gene activity in yeast. Genes Dev 20, 711722.

Mizuguchi, G., Shen, X., Landry, J., Wu, W.H., Sen, S., and Wu, C. (2004). ATPdriven exchange of histone H2AZ variant catalyzed by SWR1 chromatin remodeling complex. Science 303, 343-348. 
Ng, M.K., and Cheung, P. (2016). A brief histone in time: understanding the combinatorial functions of histone PTMs in the nucleosome context. Biochem Cell Biol 94, 33-42.

Pasini, D., Malatesta, M., Jung, H.R., Walfridsson, J., Willer, A., Olsson, L., Skotte, J., Wutz, A., Porse, B., Jensen, O.N., et al. (2010). Characterization of an antagonistic switch between histone H3 lysine 27 methylation and acetylation in the transcriptional regulation of Polycomb group target genes. Nucleic Acids Res $38,4958-4969$.

Punzeler, S., Link, S., Wagner, G., Keilhauer, E.C., Kronbeck, N., Spitzer, R.M., Leidescher, S., Markaki, Y., Mentele, E., Regnard, C., et al. (2017). Multivalent binding of PWWP2A to H2A.Z regulates mitosis and neural crest differentiation. EMBO J 36, 2263-2279.

Ruthenburg, A.J., Li, H., Patel, D.J., and Allis, C.D. (2007). Multivalent engagement of chromatin modifications by linked binding modules. Nat Rev Mol Cell Biol 8, 983-994.

Santisteban, M.S., Kalashnikova, T., and Smith, M.M. (2000). Histone H2A.Z regulats transcription and is partially redundant with nucleosome remodeling complexes. Cell 103, 411-422.

Sarcinella, E., Zuzarte, P.C., Lau, P.N., Draker, R., and Cheung, P. (2007). Monoubiquitylation of H2A.Z distinguishes its association with euchromatin or facultative heterochromatin. Molecular and cellular biology 27, 6457-6468.

Schreiber, S.L., and Bernstein, B.E. (2002). Signaling network model of chromatin. Cell 111, 771-778.

Shi, Y., Lan, F., Matson, C., Mulligan, P., Whetstine, J.R., Cole, P.A., Casero, R.A., and Shi, Y. (2004). Histone demethylation mediated by the nuclear amine oxidase homolog LSD1. Cell 119, 941-953.

Shi, Y.J., Matson, C., Lan, F., Iwase, S., Baba, T., and Shi, Y. (2005). Regulation of LSD1 histone demethylase activity by its associated factors. Molecular cell 19, 857-864.

Song, S.H., and Kim, T.Y. (2017). CTCF, Cohesin, and Chromatin in Human Cancer. Genomics Inform 15, 114-122.

Strahl, B.D., and Allis, C.D. (2000). The language of covalent histone modifications. Nature 403, 41-45.

Strubbe, G., Popp, C., Schmidt, A., Pauli, A., Ringrose, L., Beisel, C., and Paro, R. (2011). Polycomb purification by in vivo biotinylation tagging reveals cohesin and Trithorax group proteins as interaction partners. Proceedings of the National Academy of Sciences of the United States of America 108, 5572-5577. 
Subramanian, V., Fields, P.A., and Boyer, L.A. (2015). H2A.Z: a molecular rheostat for transcriptional control. F1000prime reports 7, 01.

Suganuma, T., and Workman, J.L. (2011). Signals and combinatorial functions of histone modifications. Annu Rev Biochem 80, 473-499.

Surface, L.E., Fields, P.A., Subramanian, V., Behmer, R., Udeshi, N., Peach, S.E., Carr, S.A., Jaffe, J.D., and Boyer, L.A. (2016). H2A.Z.1 Monoubiquitylation Antagonizes BRD2 to Maintain Poised Chromatin in ESCs. Cell Rep 14, 11421155.

Valdes-Mora, F., Gould, C.M., Colino-Sanguino, Y., Qu, W., Song, J.Z., Taylor, K.M., Buske, F.A., Statham, A.L., Nair, S.S., Armstrong, N.J., et al. (2017).

Acetylated histone variant H2A.Z is involved in the activation of neo-enhancers in prostate cancer. Nat Commun 8, 1346.

Van Bortle, K., and Corces, V.G. (2012). Nuclear organization and genome function. Annu Rev Cell Dev Biol 28, 163-187.

Vardabasso, C., Gaspar-Maia, A., Hasson, D., Punzeler, S., Valle-Garcia, D., Straub, T., Keilhauer, E.C., Strub, T., Dong, J., Panda, T., et al. (2015). Histone Variant H2A.Z.2 Mediates Proliferation and Drug Sensitivity of Malignant Melanoma. Molecular cell 59, 75-88.

Wang, J., Hevi, S., Kurash, J.K., Lei, H., Gay, F., Bajko, J., Su, H., Sun, W., Chang, H., Xu, G., et al. (2009a). The lysine demethylase LSD1 (KDM1) is required for maintenance of global DNA methylation. Nat Genet 41, 125-129.

Wang, Y., Zhang, H., Chen, Y., Sun, Y., Yang, F., Yu, W., Liang, J., Sun, L., Yang, X., Shi, L., et al. (2009b). LSD1 is a subunit of the NuRD complex and targets the metastasis programs in breast cancer. Cell 138, 660-672.

Zhang, H., Roberts, D.N., and Cairns, B.R. (2005). Genome-wide dynamics of Htz1, a histone H2A variant that poises repressed/basal promoters for activation through histone loss. Cell 123, 219-231. 


\section{Methods}

\section{Cell culture, transfection, plasmids, antibodies}

HEK293T cells were grown in Dulbecco's modified Eagle's medium supplemented with 10\% fetal bovine serum (DMEM; Wisent). All transfections were carried out using polyethylenimine (PEI; Polysciences). All expression constructs used were based on the pcDNA $3.1(+)$ (Invitrogen) backbone with the Flag-BirA cloned in-frame to the C-terminus of H2A.Z-1 (hereafter termed H2A.Z-FB) or H2A.Z-K3R3 where all known sites of ubiquitylation on H2A.Z is mutated to arginines. In addition, the AviTag was cloned in-frame to the $\mathrm{N}$ terminus of ubiquitin. To generate H2A.Z-FB-K3R3, K120/121/125 were mutated to R120/121/125. To generate a non-biotinylatable AviTag, K10 of the AviTag sequence (GLNDIFEAQKIEWHE) was converted to R10. In cotransfection experiments, the ratio of H2A.Z-Flag-BirA to AviTag-Ub plasmids was 3:1. Antibodies used in this study are listed in Table 1.

\section{Mononucleosome affinity purification}

Generation of mononucleosomes was performed as described previously (Draker et al., 2012). In brief, HEK293T cells were grown in $15 \mathrm{~cm}$-diameter plates and were transfected with various constructs according to the experiments. Cells were trypsinized, counted, and washed in 1X PBS, 48 hrs following transfection. Cellular pellets were resuspended in buffer A (20mM HEPES, pH 7.5, 10mM $\mathrm{KCl}, 1.5 \mathrm{mM} \mathrm{MgCl} 2,0.34 \mathrm{M}$ sucrose, $10 \%$ glycerol, $1 \mathrm{mM}$ dithiothreitol, 5mM sodium butyrate, $10 \mathrm{mM}$ NEM, and protease inhibitors), pelleted and then resuspended in buffer A containing $0.2 \%$ Triton X-100 and incubated on ice for 5 min. The nuclear suspension was centrifuged at $600 \mathrm{x}$ g; nuclei were then washed once in buffer $A$, then resuspended in cutting buffer $(15 \mathrm{mM} \mathrm{NaCl}, 60 \mathrm{mM} \mathrm{KCl}$, $10 \mathrm{mM}$ Tris $\mathrm{pH}$ 7.5, 5mM sodium butyrate, 10mM NEM, and protease inhibitors) plus $2 \mathrm{mM} \mathrm{CaCl}_{2}$. Microccocal nuclease (MNase; Worthington) was added at a concentration of 10 units $/ 1.0 \times 10^{7}$ cells then incubated at $37^{\circ} \mathrm{C}$ for $30 \mathrm{~min}$. The reaction was stopped by the addition of $20 \mathrm{mM}$ EGTA (one twenty-fifth of the reaction volume) and immediate gentle mixing by inversion. The MNase- 
digested nuclei were centrifuged at $1300 \times \mathrm{g}$. The resulting supernatant (S1) was saved and kept on ice. The digested nuclear pellet was subjected to hypotonic lysis by resuspension in TE buffer (10mM Tris-HCL, pH 8.0, 1mM EDTA). Samples were incubated on ice for $1 \mathrm{hr}$, with occasional mixing by pipette. The suspension was then centrifuged at $16000 \times \mathrm{g}$ and the supernatant (S2) was transferred to a new tube. Salt was adjusted in $\mathrm{S} 1$ to $150 \mathrm{mM} \mathrm{NaCl}$ by adding $2 \mathrm{X}$ buffer D (30 mM Tris pH 7.5, $225 \mathrm{mM} \mathrm{NaCl}, 3 \mathrm{mM} \mathrm{MgCl}$, 20\% glycerol, $0.4 \%$ Triton-X 100, 5mM sodium butyrate, 10mM NEM, and protease inhibitors) dropwise, with constant mixing on a vortex set to low speed. S2 was also titrated to $150 \mathrm{mM} \mathrm{NaCl}$ by the drop-wise addition of 3X buffer E (60 mM HEPES pH 7.5, $450 \mathrm{mM} \mathrm{NaCl}, 4.5$ mM MgCl2, 0.6 mM EGTA, 0.6 \% Triton-X 100, 30\% glycerol, $5 \mathrm{mM}$ sodium butyrate, $10 \mathrm{mM}$ NEM, and protease inhibitors). Insoluble material was pelleted via centrifugation. The clarified supernatants were combined and then used for affinity purification. Streptavidin-agarose (Sigma) or Flag M2agarose beads (Sigma) were added and incubated overnight at $4^{\circ} \mathrm{C}$ on an endover-end rotator. Beads were washed 4 times in $1 X$ Buffer D, followed by 3 washes in 1X Buffer D containing 0.5\% Triton X-100. Proteins were eluted from the beads by resuspension in 2 X SDS sample buffer and boiled for $10 \mathrm{~min}$. For Western blot analysis, samples were run on SDS-polyacrylamide electrophoresis gels according to standard practices.

\section{ChIP-Sequencing}

Mononucleosome affinity purification was performed in duplicate as described above using streptavidin-agarose, Flag M2-agarose, or H3 antibody (pulleddown using protein G-coupled Dynabeads; Invitrogen) and eluted in buffer D containing $1 \%$ SDS by end-over-end rotation at room temperature for 2 X $10 \mathrm{~min}$. DNA was treated with RNase A and proteinase K, purified by phenolchloroform extraction, and then re-precipitated with ethanol and resuspended in water. DNA was converted to libraries using Illumina TruSeq ChIP-Seq and sequenced on an Illumina NextSeq500 in single end mode. Reads were converted to FASTQ format and mapped to the human hg19 genome using Bowtie. Duplicate reads were removed. Peaks were called using MACS2 using H3 as a control. Normalized peaks from duplicate experiments were then averaged and 
matched H3 samples were subtracted to yield fragments per million reads (FPM). The IDR procedure employed by ENCODE was then used to generate a merged peak set for both streptavidin and Flag with all raw peaks as input. At FDR $<0.05$, 143k peaks (from 453k and 552k in streptavidin or Flag samples, respectively) were recovered in both. Enhancers were identified as colocalization of H3K4me1, H3K27ac, and DNaseI hypersensitivity sites (HSS) in either HeLa or several other cells and tissues.

\section{ChIP-qPCR}

Affinity-purified mononucleosomes were eluted in buffer D-1\% SDS as described above. DNA was treated with RNase A and proteinase $\mathrm{K}$, phenol-chloroform extracted from mononucleosomes, re-precipitated with ethanol, and then resuspended in water. Quantitative polymerase chain reactions (qPCR) were assembled in triplicate using PerfeCta SYBR Green SuperMix (Quanta Biosciences) and gene-specific primers. Reactions were run on an Opticon 2 thermocycler (Biorad). Primers used are listed in Table 2. 


\section{$\mathrm{Ng}$ et al, Figure 1}

A

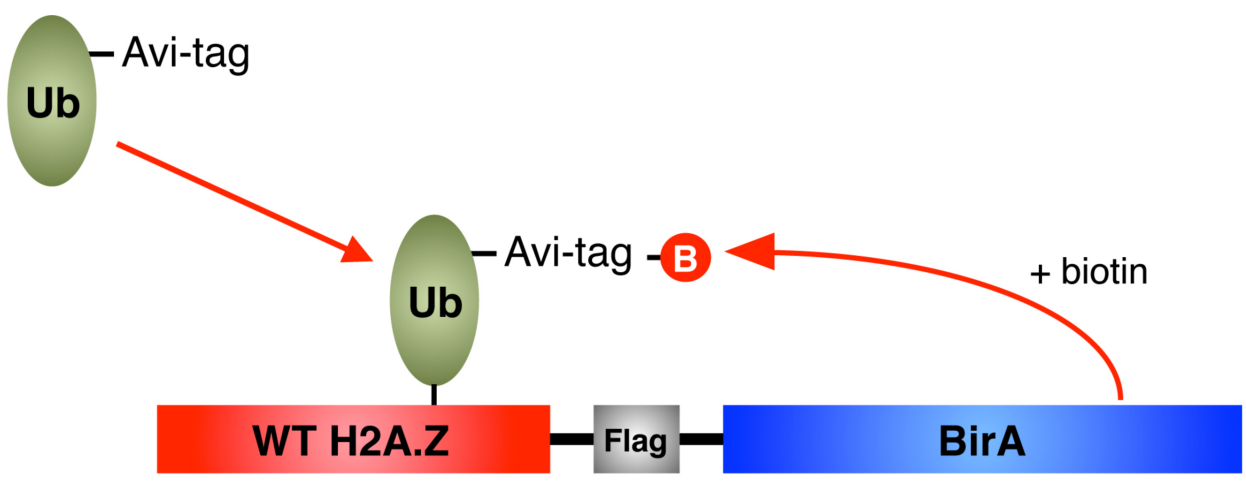

B

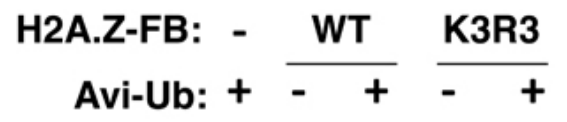
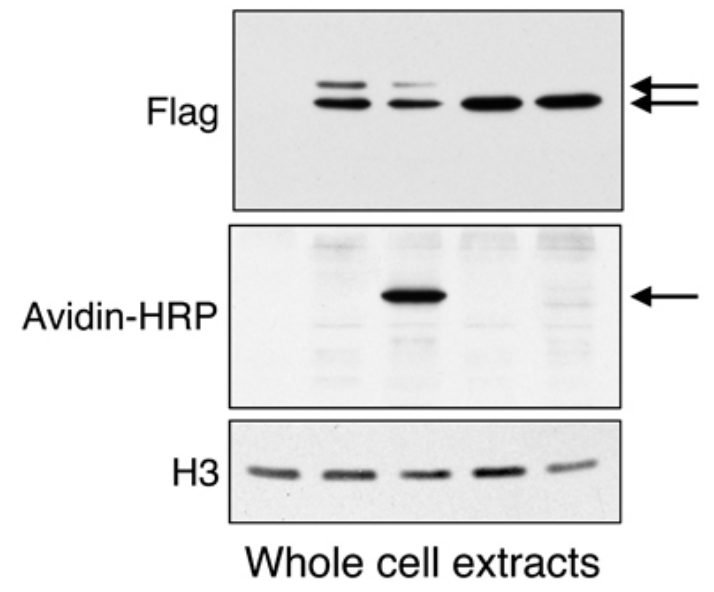

$\begin{aligned} & \text { H2A.Z-FB: }- \text { WT } \\ & \text { Avi-Ub: }+\frac{\text { K3R3 }}{-}+\frac{+}{-}\end{aligned}$

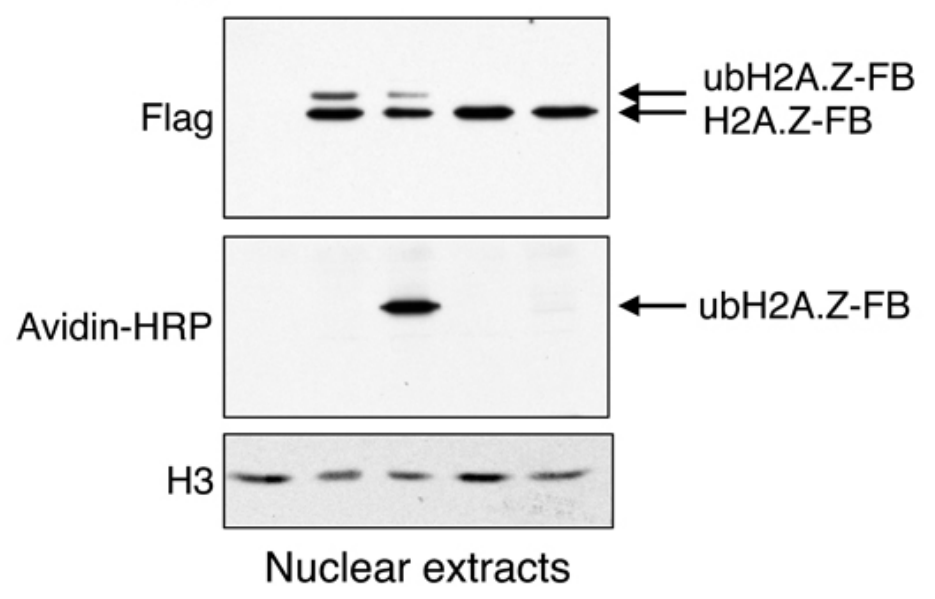

C

\section{Mono-nucleosome fraction}

Flag-BirA: NLS - NLS H2A.Z WT H2A.Z KR

Avi-Ub: $-++\overline{-}++$ Mw
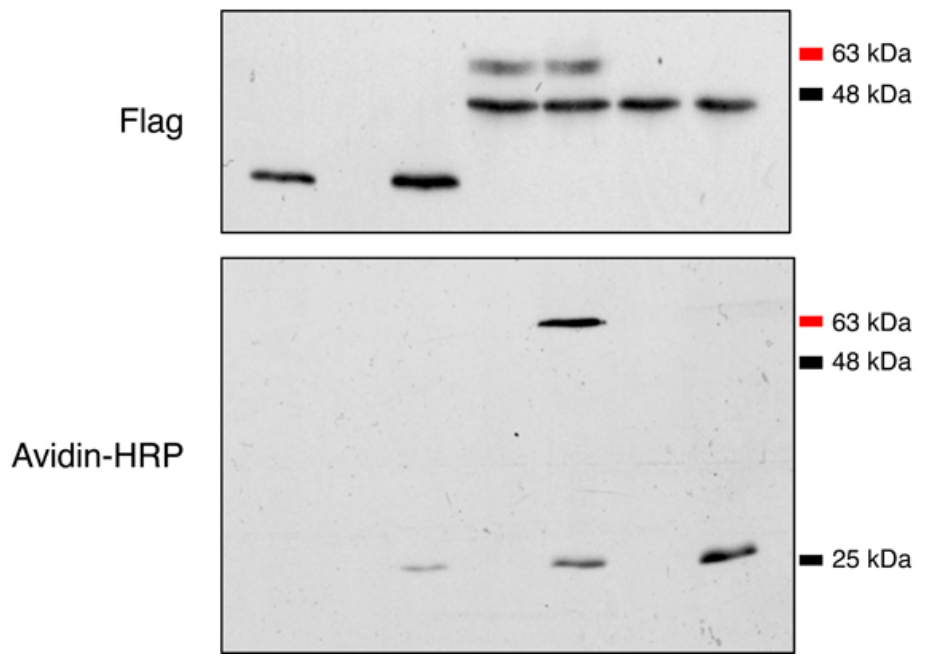
A

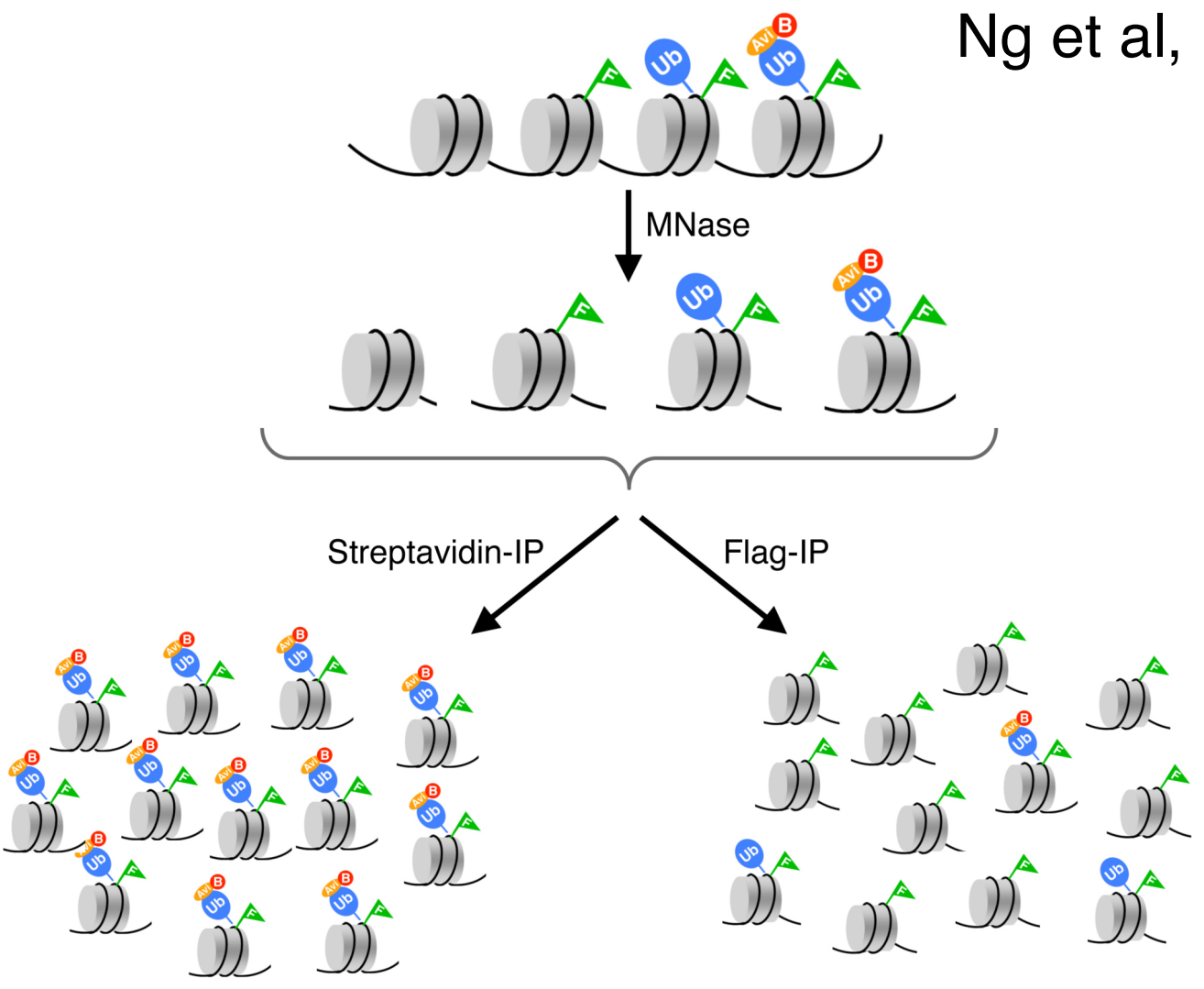

B

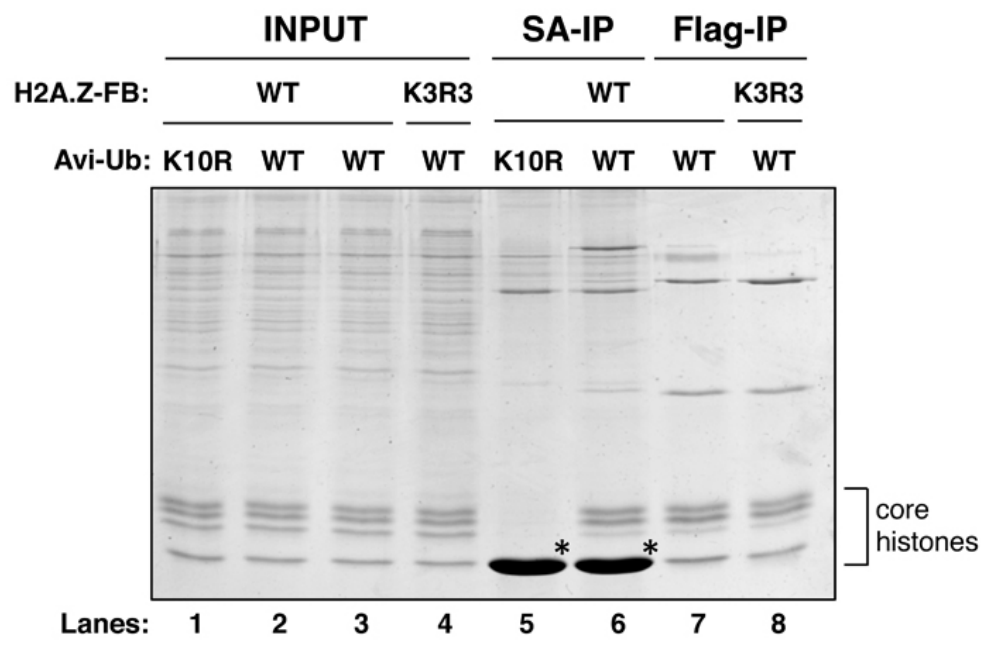

C

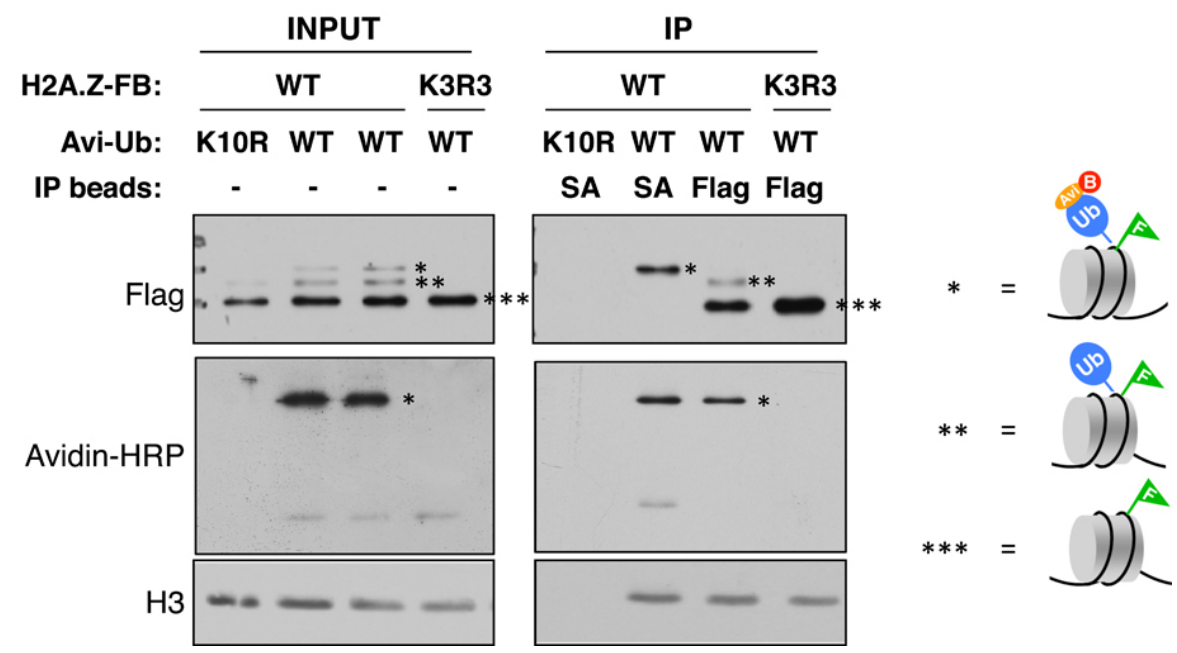




\section{$\mathrm{Ng}$ et al, Figure 3}
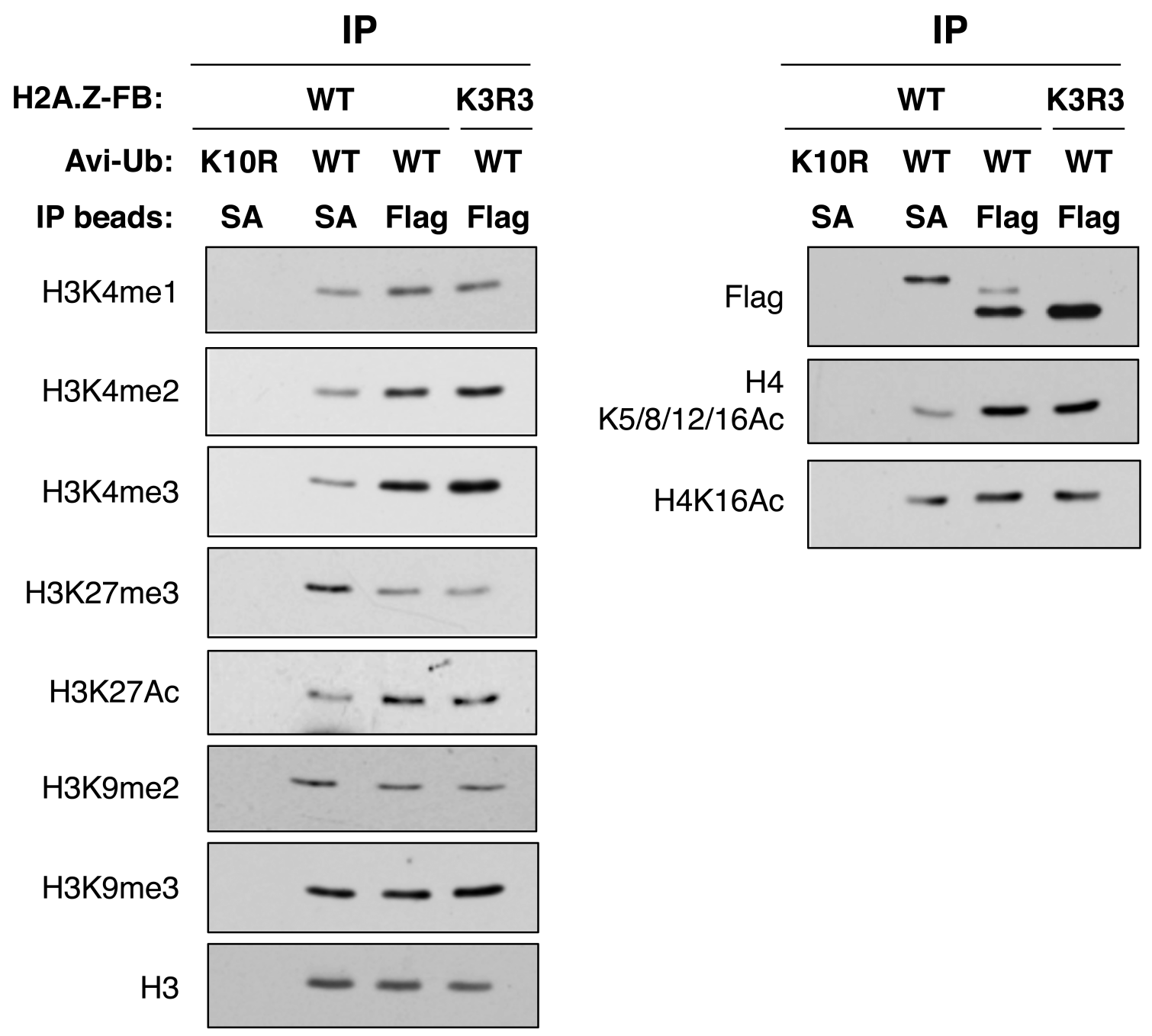
bioRxiv preprint doi: https://doi.org/10.1101/759852; this version posted October 31, 2019. The copyright holder for this preprint (which was not certified by peer review) is the author/funder. All rights reserved. No reuse allowed without permission.

A

Avi

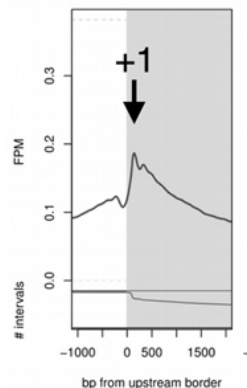

B

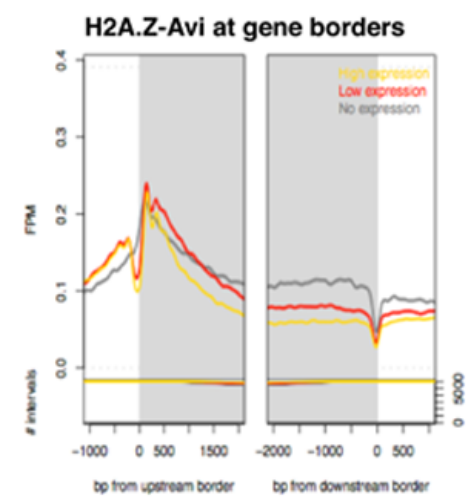

C
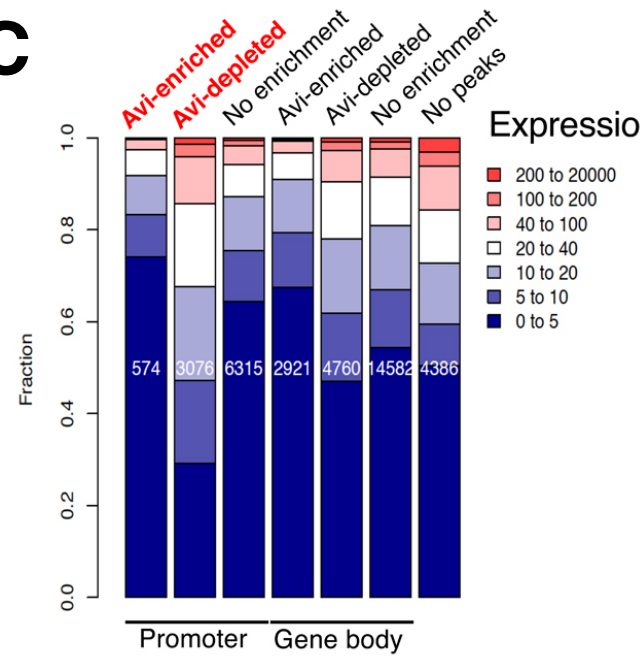

E

Cluster density of Avi-enriched H2A.Z across genomic regions:

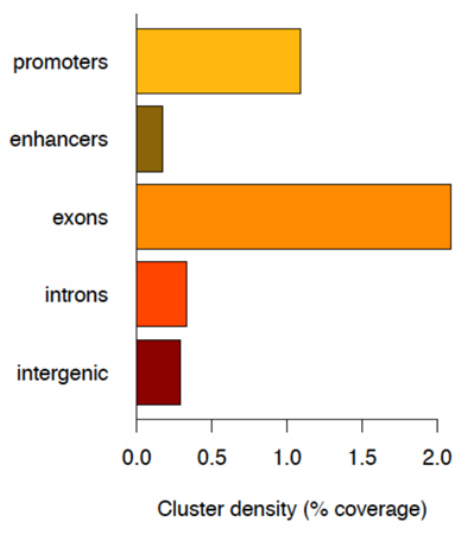

$\mathrm{Ng}$ et al, Figure 4

FLAG

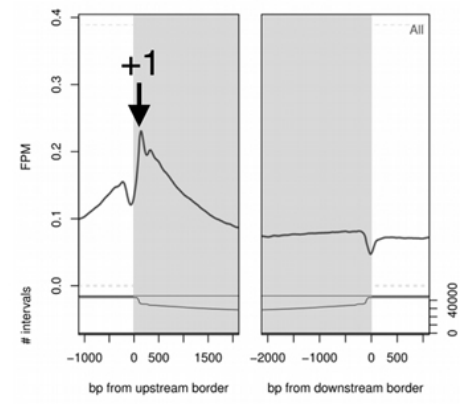

Flag-normalized Avi at gene borders
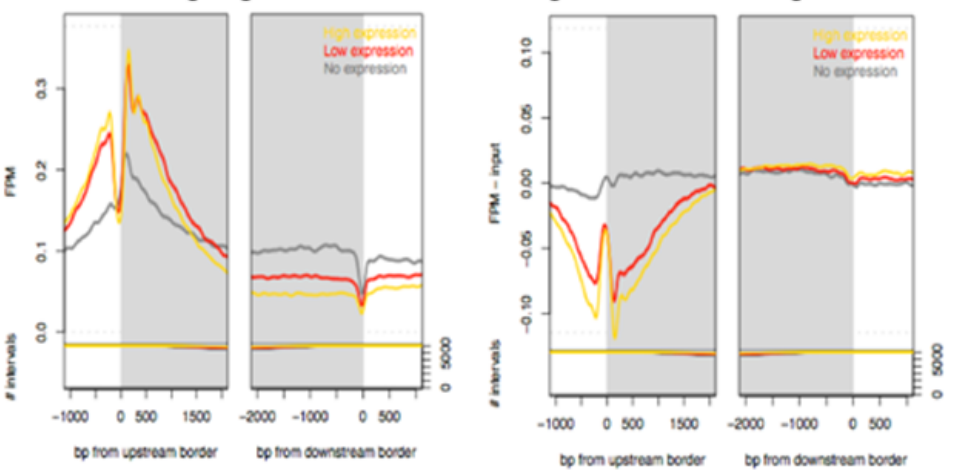

D

Fraction of tissues/cell types with $>$ average expression

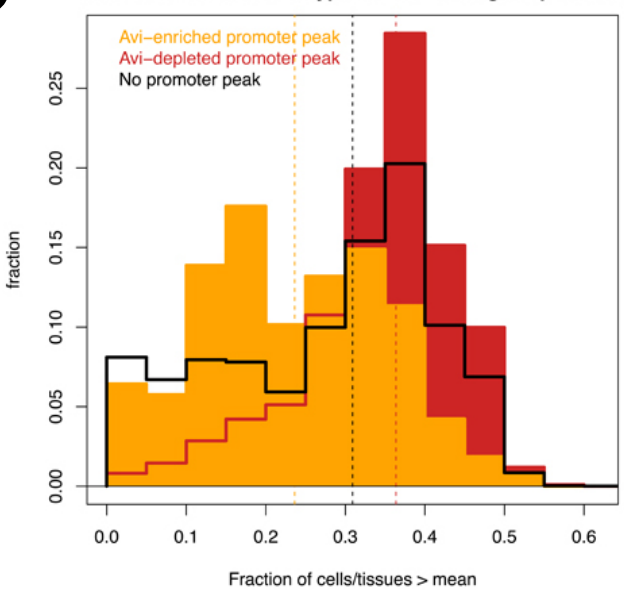

Cluster density of Avi-depleted H2A.Z across genomic regions:

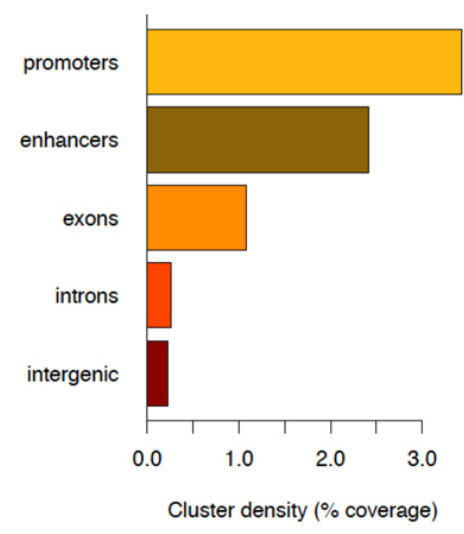


bioRxiv preprint doi: https://doi.org/10.1101/759852; this version posted October 31, 2019. The copyright holder for this preprint (which was not certified by peer review) is the author/funder. All rights reserved. No reuse allowed without permission.

\section{$\mathrm{Ng}$ et al, Figure 5}

A

SA/FLAG

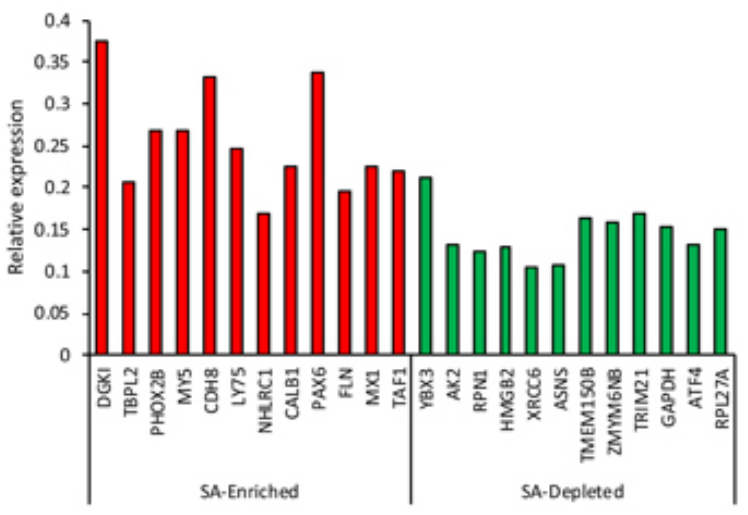

$\mathrm{H} 3 \mathrm{~K} 4 \mathrm{me} 3 / \mathrm{H} 3$

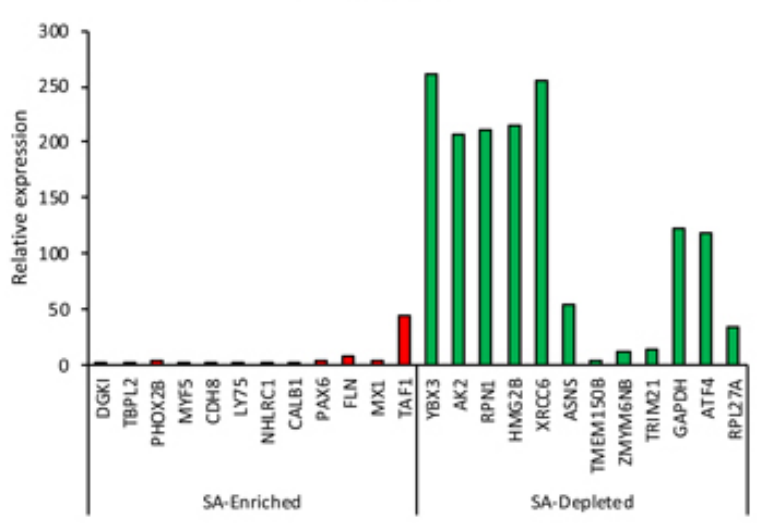

B

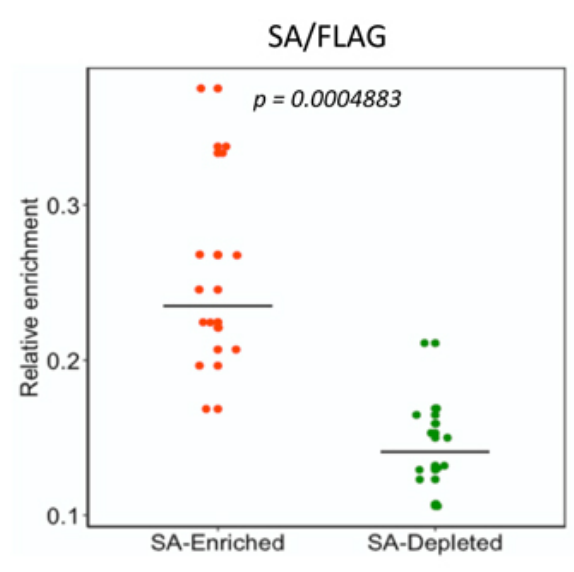

$\mathrm{H} 3 \mathrm{~K} 4 \mathrm{me} 3 / \mathrm{H3}$

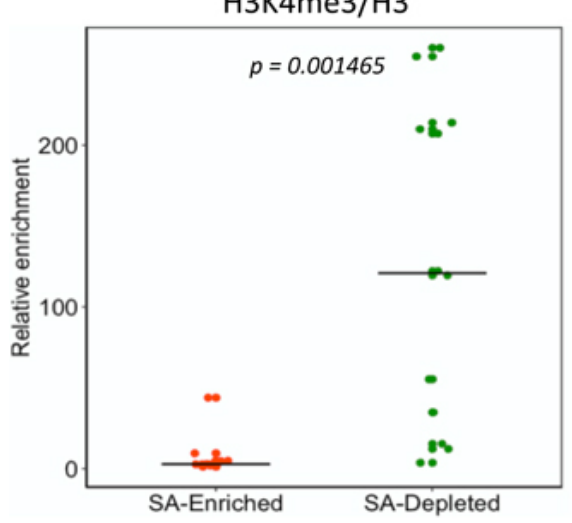

H2A.Z/H3

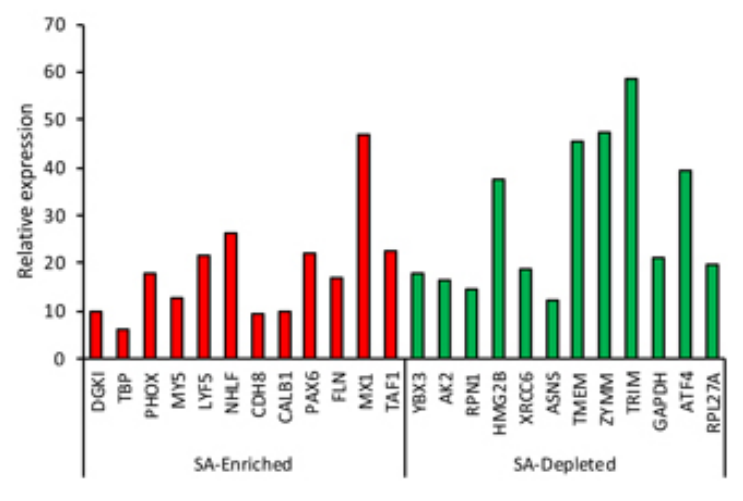

$\mathrm{H} 3 \mathrm{~K} 27 \mathrm{me} 3 / \mathrm{H3}$
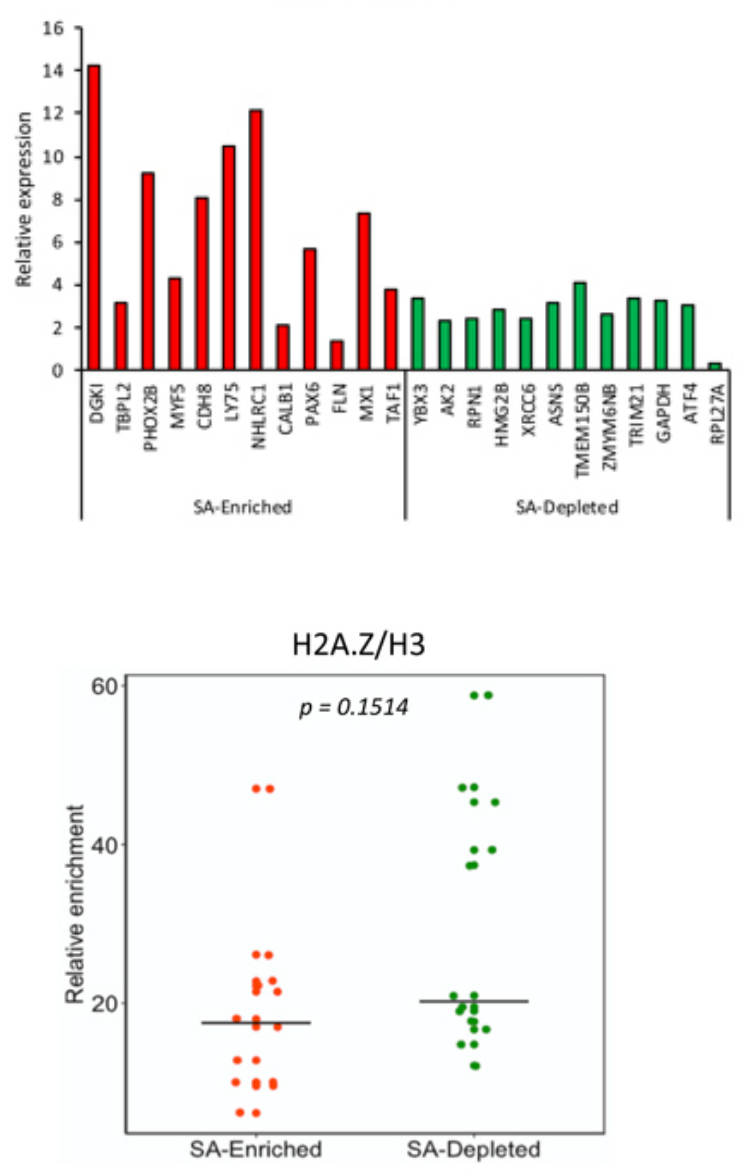

H3K27me3/H3

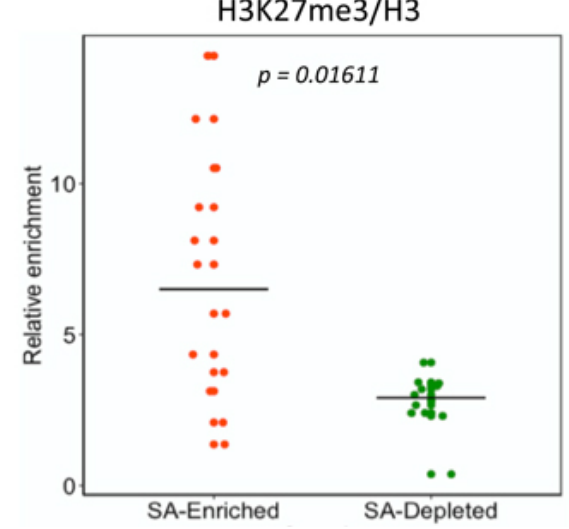




\section{$\mathrm{Ng}$ et al, Figure 6}

A
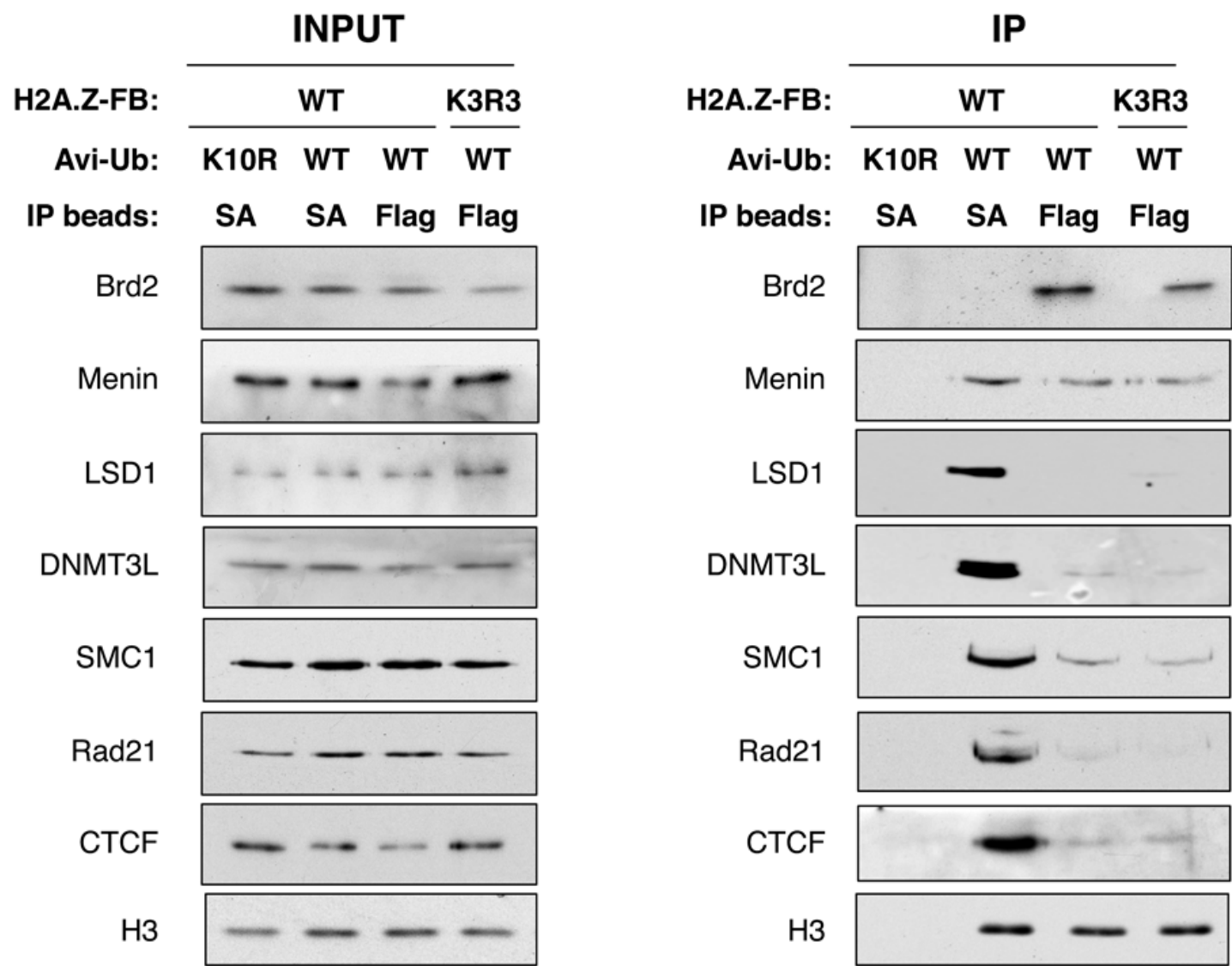

B

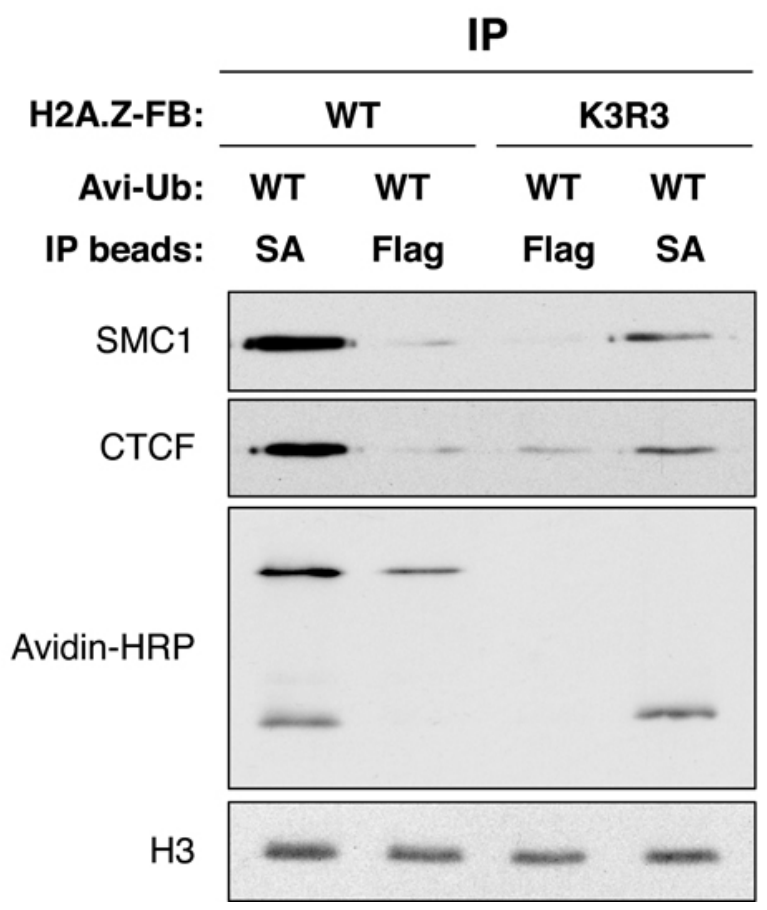

